\title{
Ratio asymptotics for polynomials orthogonal on arcs of the unit circle
}

\author{
D. Barrios Rolanía \\ Universidad Politécnica de Madrid \\ Spain
}

\author{
G. López Lagomasino* \\ Universidad Carlos III de Madrid \\ Spain
}

\begin{abstract}
Ratio asymptotics for orthogonal polynomials on the unit circle is characterized in terms of the existence of $\lim _{n}\left|\Phi_{n}(0)\right|$ and $\lim _{n} \frac{\Phi_{n+1}(0)}{\Phi_{n}(0)}$, where $\left\{\Phi_{n}(0)\right\}_{n \geq 0}$ denotes the sequence of reflection coefficients. The limit periodic case, that is when these limits exist for $n=j \bmod k, j=1, \ldots, k$, is also considered.

Keywords: Orthogonal polynomials, ratio asymptotics, reflection coefficients.

AMS Subject classification: Primary 42C05.
\end{abstract}

\section{Introduction}

1. The class of finite Borel measures supported on the real line, whose sequence of orthogonal polynomials satisfy ratio asymptotics, has been characterized in terms of the limit behavior of their recurrence coefficients. This is the so called Blumenthal-Nevai class (see [16]). (In particular, the support of such measures consists of a segment plus at most a denumerable set of points which may only accumulate on the extreme points of the segment.) The main purpose of this paper is to obtain the corresponding result for the case of measures supported on the unit circle $\Gamma$. Some important steps have already been taken.

In [12] there is a spectral theoretic approach to the proof of the following theorem of Ya. L. Geronimus (see [7]):

Let $\mu$ be a probability measure on $\Gamma$ and let $\left\{\Phi_{n}\right\}_{n \geq 0}$, be the corresponding sequence of monic orthogonal polynomials. Suppose that

$$
\lim _{n \longrightarrow \infty} \Phi_{n}(0)=a \quad, \quad 0<|a|<1 .
$$

Set $\sin \frac{\alpha}{2}:=|a|, \alpha \in(0, \pi)$ and $\gamma_{\alpha}:=\left\{e^{i \theta}: \alpha \leq \theta \leq 2 \pi-\alpha\right\}$. Then $\gamma_{\alpha} \subseteq \operatorname{supp}(\mu)$ and $\operatorname{supp}(\mu) \backslash \gamma_{\beta}$ is finite for every $0<\beta<\alpha$.

${ }^{*}$ The research of this author was partially supported by Dirección General de Enseñanza Superior under grant PB 96-0120-C03-01 and by INTAS under grant 93-0219 EXT. 
The proof of this result (see Theorem 3 in [12]) follows through if instead of (1) we require the weaker conditions

$$
\lim _{n \longrightarrow \infty}\left|\Phi_{n}(0)\right|=a \in(0,1], \lim _{n \longrightarrow \infty} \frac{\Phi_{n+1}(0)}{\Phi_{n}(0)}=b .
$$

In honor of truth, we should say that Geronimus' proof, using continued fractions, adapts as well to this apparently naive extension of his result.

In [4] it was shown:

Let $\operatorname{supp}(\mu)=\gamma$, where $\gamma$ is an arc contained in the unit circle $\Gamma$ and let $\mu^{\prime}>0$ a.e on $\gamma$, then

$$
\lim _{n \longrightarrow \infty} \frac{\Phi_{n+1}(z)}{\Phi_{n}(z)}=C(\gamma) G(z)
$$

uniformly on compact subsets of $\mathbb{C} \backslash \gamma$ and

$$
\lim _{n \longrightarrow \infty} \frac{\kappa_{n+1}}{\kappa_{n}}=\frac{1}{C(\gamma)}
$$

where $C(\gamma)$ denotes the logarithmic capacity of $\gamma, G(z)$ the conformal mapping of $\overline{\mathbb{C}} \backslash \gamma$ onto $[|w|>1]$ such that $G(\infty)=\infty$ with $G^{\prime}(\infty)>0$ and

$$
\kappa_{n}=\left\{\int\left|\Phi_{n}(z)\right|^{2} d \mu(z)\right\}^{-1 / 2} .
$$

This result extends one of E. A. Rakhmanov given for the case when $\mu^{\prime}>0$ a.e. on all $\Gamma$ (see [21]-[22] for the original proof, also [15] and [23] for simpler ones).

From (3)-(4) immediately follows (2) with $b=C(\gamma) G(0)$ and $a=\sqrt{1-C^{2}(\gamma)}$ but, in general, (1) doesn't take place. Thus, we conclude that (2) should be the characterization, in terms of the reflection coefficients, of those measures on the unit circle whose orthogonal polynomials have ratio asymptotics. In case of the real line a measure $\lambda$ with compact support $[c, d]$ such that $\lambda^{\prime}>0$ a.e. on $[c, d]$ must be in the Blumenthal-Nevai class (see [21] - [22]). Our first reaction was to check that Geronimus' Theorem could be extended to this class of measures.

2. In fact, we shall consider a wider class of measures.

Definition 1. We say that $\mu \in M_{\Gamma}\left(a_{1}, \ldots, a_{k} ; b_{1}, \ldots, b_{k}\right)$ if for all $j=1, \ldots, k$, we have

$$
\lim _{n=j \bmod k}\left|\Phi_{n}(0)\right|=a_{j}, \lim _{n=j \bmod k} \frac{\Phi_{n}(0)}{\Phi_{n-1}(0)}=b_{j} .
$$

We shall restrict our attention to probability Borel measures on the unit circle. For arbitrary finite Borel measures the corresponding results are easily deduced. For simplicity, we limit ourselves in the introduction to the case when $k=1$. Then (5) reduces to (2) and we write $M_{\Gamma}(a ; b)$. For any sequence of orthogonal polynomials on the unit circle, we have that $\left|\Phi_{n}(0)\right|<1$. Therefore, if the first limit in (2) exists then necessarily $a \in[0,1]$. When $a=0$, the values admissible for $b$ are all those with $|b| \leq 1$. In this situation, it is well known (even if the second limit in (2) doesn't exist) that there is ratio asymptotics of type (3)-(4) outside and on the unit circle with $C(\gamma)=1$ (the capacity of the whole unit circle) and $G(z)=z$ (the conformal mapping of $[|z|>1]$ onto $[|w|>1]$ such that $G(\infty)=\infty$ and $\left.G^{\prime}(\infty)>0\right)$. When $|b|<1$ the measure not only belongs to the Szegö 
class but it is also absolutely continuous with respect to the Lebesgue measure (see [10], Theorem 8.5, p. 163) and it is to be expected that ratio asymptotics extends uniformly on all compact subsets of $[|z|>|b|]$. In Theorem 1, we consider that $a \in(0,1]$, thus Szegö's condition is not satisfied and $|b|=1$. Set

$$
\gamma=\{z \in \Gamma: \alpha \leq \arg (z)-\arg (b) \leq 2 \pi-\alpha\},
$$

where $\sin \frac{\alpha}{2}:=a$. We prove:

Theorem 1. Let $a \in(0,1]$. The following conditions $i)$ and ii) are equivalent:

i) $\mu \in M_{\Gamma}(a ; b)$,

ii)

$$
\lim _{n \longrightarrow \infty} \frac{\Phi_{n+1}(z)}{\Phi_{n}(z)}=\frac{1}{2}\left[(z+b)+\sqrt{(z-b)^{2}+4 z b a^{2}}\right], \lim _{n \longrightarrow \infty} \frac{\kappa_{n}}{\kappa_{n+1}}=\sqrt{1-a^{2}}
$$

where convergence is uniform on each compact subset of $\mathbb{C} \backslash \operatorname{supp}(\mu)$ and the root is taken so that $\sqrt{1}=1$. Under any one of the conditions above, we have:

iii) the derived set of $\operatorname{supp}(\mu)$ equals $\gamma$,

iv) for any compact subset $K$ contained in $\mathbb{C} \backslash \operatorname{supp}(\mu)$ and for all sufficiently large $n$ the polynomials $\Phi_{n}(z)$ have no zeros on $K$,

$v)$ let $\zeta$ be an isolated mass point in $\operatorname{supp}(\mu)$; then for all sufficiently small $r>0$, there exists $n_{0}$ such that for $n \geq n_{0}, \Phi_{n}(z)$ has exactly one zero in $\{z:|z-\zeta| \leq r\}$.

$v i)$

$$
\lim _{n \longrightarrow \infty} \frac{\Phi_{n+1}(z)}{\Phi_{n}(z)}=\frac{1}{2}\left[(z+b)-\sqrt{(z-b)^{2}+4 z b a^{2}}\right], z \in \operatorname{supp}(\mu) \backslash \gamma
$$

In the proof of Theorem 1, we use a scheme similar to the one employed in [1] (see also [2], [3]) where we studied the convergenge of diagonal Padé approximants (J-fractions) and an extension of the Blumenthal-Nevai class to the case when the recurrence coefficients are allowed to take complex values.

3. The paper is divided as follows. In section 2, we introduce the necessary notation and prove several auxiliary lemmas. The key results, Theorems 2 and 3 are proved in Section 3. From these theorems the main statements of Theorem 1 , for $a \in(0,1)$, are an immediate consequence. Theorems 2 and 3 involve measures in $M_{\Gamma}\left(a_{1}, \ldots, a_{k} ; b_{1}, \ldots, b_{k}\right)$ and ratio asymptotics of their orthogonal polynomials. Section 3 also contains some statements (see Theorem 4) regarding the rational approximation of Caratheodory functions in the complement of the support of the corresponding measure when it is in the class prescribed above. The final section 4 is dedicated to some corollaries of the main results. In its second part, a sketch of the proof of Theorem 1 for $a=1$ is given.

\section{Notation and Auxiliary Results}


1. As above $\mu$ is a probability measure with infinite $\operatorname{support} \operatorname{supp}(\mu) \subset \Gamma:=\{z:|z|=1\}$, $\left\{\Phi_{n}(z)\right\}_{n \geq 0}$ the corresponding sequence of monic orthogonal polynomials (deg $\Phi_{n}=n$ ) and $\kappa_{n}=\left\{\int\left|\Phi_{n}(z)\right|^{2} d \mu(z)\right\}^{-1 / 2}$. The complex numbers $\Phi_{n}(0), n \geq 0$, are known as reflection coefficients. They play an important role in the description of the polynomials and their asymptotic properties. In particular, it is well known that the orthonormal polynomials $\phi_{n}=\kappa_{n} \Phi_{n}, n \geq 0$, satisfy the following relations:

$$
\sum_{i=0}^{n-1} \frac{\kappa_{i}}{\kappa_{n}} \Phi_{n+1}(0) \overline{\Phi_{i}(0)} \phi_{i}(z)+\left(z+\Phi_{n+1}(0) \overline{\Phi_{n}(0)}\right) \phi_{n}(z)-\frac{\kappa_{n}}{\kappa_{n+1}} \phi_{n+1}(z)=0, \quad n \geq 1
$$

where $\kappa_{m}$ and the reflection coefficients are related by

$$
\frac{\kappa_{m-1}}{\kappa_{m}}=\sqrt{1-\left|\Phi_{m}(0)\right|^{2}}, \kappa_{m}^{2}=\frac{1}{\prod_{i=1}^{m}\left(1-\left|\Phi_{i}(0)\right|^{2}\right)}, m \geq 1, \quad \kappa_{0}=1
$$

(see [12]).

Another important tool in the research of these polynomials is the multiplication operator $U$ on $L^{2}(\mu)$ given by

$$
(U f)(z)=z f(z)
$$

for each $f \in L^{2}(\mu)$, where $L^{2}(\mu)$ is the space of square integrable functions with respect to $\mu$. If $\log \mu^{\prime} \notin L^{1}(\mu)$ (that is, if Szegö's condition is not satisfied), then the orthogonal polynomials are dense in $L^{2}(\mu)$ and the sequence $\left\{\phi_{n}\right\}_{n \geq 0}$ is an orthonormal basis of $L^{2}(\mu)$ (see [10] pp 14). In this case, the infinite Hessenberg matrix

$$
U=U(\mu)=\left(\begin{array}{cccc}
-\Phi_{1}(0) \overline{\Phi_{0}(0)} & -\frac{\kappa_{0}}{\kappa_{1}} \Phi_{2}(0) \overline{\Phi_{0}(0)} & -\frac{\kappa_{0}}{\kappa_{2}} \Phi_{3}(0) \overline{\Phi_{0}(0)} & \ldots \\
\frac{\kappa_{0}}{\kappa_{1}} & -\Phi_{2}(0) \overline{\Phi_{1}(0)} & -\frac{\kappa_{1}}{\kappa_{2}} \Phi_{3}(0) \overline{\Phi_{1}(0)} & \ldots \\
0 & \frac{\kappa_{1}}{\kappa_{2}} & -\Phi_{3}(0) \overline{\Phi_{2}(0)} & \ldots \\
0 & 0 & \frac{\kappa_{2}}{\kappa_{3}} & \ldots \\
\vdots & \vdots & \vdots & \ddots
\end{array}\right)
$$

(where $\Phi_{0}(0)=1$ ) is the matrix representation of the unitary operator (9) in the basis $\left\{\phi_{n}\right\}_{n \geq 0}$ (compare (7) and (9)). In terms of the monic orthogonal polynomials (7) may be rewritten as

$$
\Phi_{n+1}(z)=\left(z+\Phi_{n+1}(0) \overline{\Phi_{n}(0)}\right) \Phi_{n}(z)+\sum_{i=0}^{n-1}\left(\frac{\kappa_{i}}{\kappa_{n}}\right)^{2} \Phi_{n+1}(0) \overline{\Phi_{i}(0)} \Phi_{i}(z), \quad n \geq 1 .
$$

Let $U_{n}$ be the principal section of $U$ of order $n$. Expanding $\operatorname{det}\left(z I_{n}-U_{n}\right)$ in terms of the last column, from (11) it is easy to see that

$$
\Phi_{n}(z)=\operatorname{det}\left(z I_{n}-U_{n}\right), \quad n=0,1, \ldots
$$

(taking $\left.\operatorname{det}\left(z I_{0}-U_{0}\right)=1\right)$.

Consider the column vector

$$
u=\left(\phi_{0}(z), \phi_{1}(z), \ldots, \phi_{n}(z), \ldots\right)^{T} .
$$


Formula (7) conjugated indicates that the formal matrix operation of the adjoint $U^{*}$ of $U$ on $\bar{u}$ gives

$$
U^{*} \bar{u}=\bar{z} \bar{u}
$$

This and the remarks above give:

Lemma 1. Let $\mu$ be a probability measure for which Szegö's condition is not satisfied, then

$$
\operatorname{supp}(\mu)=\sigma(U)
$$

and $z \in \sigma_{p}(U)$ if and only if $u \in l^{2}$, where $\sigma(U)$ and $\sigma_{p}(U)$ denote the spectrum and the point spectrum of $U$, respectively.

It is well known, but not very frequently used, that the orthogonal polynomials on the unit circle satisfy a three-term recurrence relation. For completeness, we include a proof.

Lemma 2. We have

$$
\left.\begin{array}{r}
\Phi_{0}(0) \frac{\kappa_{0}}{\kappa_{1}} \phi_{1}(z)-\left(z \Phi_{0}(0)+\Phi_{1}(0)\right) \phi_{0}(z)=0, \\
\Phi_{n}(0) \frac{\kappa_{n}}{\kappa_{n+1}} \phi_{n+1}(z)-\left(z \Phi_{n}(0)+\Phi_{n+1}(0)\right) \phi_{n}(z)+z \frac{\kappa_{n-1}}{\kappa_{n}} \Phi_{n+1}(0) \phi_{n-1}(z)=0, \\
n \in \mathbb{N} .
\end{array}\right\}
$$

Proof. From (11), we have that

$$
\frac{\Phi_{n+1}(0)}{\kappa_{n}^{2}} \sum_{i=0}^{n-1} \kappa_{i}^{2} \overline{\Phi_{i}(0)} \Phi_{i}(z)+\left(z+\Phi_{n+1}(0) \overline{\Phi_{n}(0)}\right) \Phi_{n}(z)-\Phi_{n+1}(z)=0, \quad n \in \mathbb{N} .
$$

Thus,

$$
\frac{\Phi_{n}(0)}{\kappa_{n-1}^{2}} \sum_{i=0}^{n-2} \kappa_{i}^{2} \overline{\Phi_{i}(0)} \Phi_{i}(z)+\left(z+\Phi_{n}(0) \overline{\Phi_{n-1}(0)}\right) \Phi_{n-1}(z)-\Phi_{n}(z)=0, \quad n \geq 2 .
$$

Multiplying in (14) by $\frac{\Phi_{n}(0)}{\kappa_{n-1}^{2}}$ and using (15), we obtain

$$
\begin{aligned}
0 & =\frac{\Phi_{n+1}(0)}{\kappa_{n}^{2}}\left[\frac{\Phi_{n}(0)}{\kappa_{n-1}^{2}} \sum_{i=0}^{n-2} \kappa_{i}^{2} \overline{\Phi_{i}(0)} \Phi_{i}(z)+\Phi_{n}(0) \overline{\Phi_{n-1}(0)} \Phi_{n-1}(z)\right] \\
& +\frac{\Phi_{n}(0)}{\kappa_{n-1}^{2}}\left(z+\Phi_{n+1}(0) \overline{\Phi_{n}(0)}\right) \Phi_{n}(z)-\frac{\Phi_{n}(0)}{\kappa_{n-1}^{2}} \Phi_{n+1}(z) \\
& =\frac{\Phi_{n+1}(0)}{\kappa_{n}^{2}}\left[\Phi_{n}(z)-z \Phi_{n-1}(z)\right]+\frac{\Phi_{n}(0)}{\kappa_{n-1}^{2}} z \Phi_{n}(z) \\
& +\frac{\Phi_{n+1}(0)\left|\Phi_{n}(0)\right|^{2}}{\kappa_{n-1}^{2}} \Phi_{n}(z)-\frac{\Phi_{n}(0)}{\kappa_{n-1}^{2}} \Phi_{n+1}(z), n \geq 2,
\end{aligned}
$$

which, using (8), reduces to

$$
\Phi_{n}(0) \Phi_{n+1}(z)-\left(z \Phi_{n}(0)+\Phi_{n+1}(0)\right) \Phi_{n}(z)+z \frac{\kappa_{n-1}^{2}}{\kappa_{n}^{2}} \Phi_{n+1}(0) \Phi_{n-1}(z)=0, n \geq 2 .
$$


For $n=1$, (16) follows directly from (15). Multipliying (16) by $\kappa_{n}$, we arrive to (13) (for $n \in \mathbb{N}$ ). The first relation in (13) is trivial.

In the sequel, for simplicity, we shall assume that $\Phi_{n}(0) \neq 0, n \in \mathbb{N}$. Nevertheless, we make no explicit reference to this restriction in the statements of our main results because it is easy to adjust the arguments used in their proof if only $\Phi_{n}(0) \neq 0$ for all sufficiently large $n$. This is always the case when $\mu \in M_{\Gamma}\left(a_{1}, \ldots, a_{k} ; b_{1}, \ldots, b_{k}\right)$ and $a_{i} \neq 0, i=1, \ldots, k$.

The three-term recurrence relation motivates our interest in the following tri-diagonal matrix. Set

$$
D(z)=\left(\begin{array}{cccc}
z+\frac{\Phi_{1}(0)}{\Phi_{0}(0)} & z \frac{\Phi_{2}(0)}{\Phi_{1}(0)}\left(\frac{\kappa_{0}}{\kappa_{1}}\right)^{2} & 0 & \ldots \\
1 & z+\frac{\Phi_{2}(0)}{\Phi_{1}(0)} & z \frac{\Phi_{3}(0)}{\Phi_{2}(0)}\left(\frac{\kappa_{1}}{\kappa_{2}}\right)^{2} & \ldots \\
0 & 1 & z+\frac{\Phi_{3}(0)}{\Phi_{2}(0)} & \ldots \\
\vdots & \vdots & \vdots & \ddots
\end{array}\right) .
$$

By $D^{(m)}(z)$ we denote the infinite tri-diagonal matrix obtained after eliminating from $D(z)$ the first $m$ rows and $m$ columns $\left(D^{(0)}(z)=D(z)\right) . D_{n}^{(m)}(z)$ denotes the principal section of order $n$ of $D^{(m)}(z)$. Define

$$
\Delta_{n}^{(m)}(z)=\operatorname{det}\left(D_{n}^{(m)}(z)\right), \Delta_{0}^{(m)}(z) \equiv 1 .
$$

Lemma 3. We have

$$
\Phi_{n}(z)=\Delta_{n}^{(0)}(z), \quad n \geq 0
$$

Proof. Expanding $\Delta_{n}^{(0)}(z)$ by the last column, we see that $\left\{\Delta_{n}^{(0)}(z)\right\}_{n \geq 0}$, satisfies the same three-term recurrence relation as $\left\{\Phi_{n}(z)\right\}_{n \geq 0}$ (see (16)). On the other hand, $\Delta_{0}^{(0)}(z) \equiv$ $1 \equiv \Phi_{0}(z)$ and $\Delta_{1}^{(0)}(z)=z+\Phi_{1}(0)=\Phi_{1}(z)$. Since the initial conditions coincide so do the sequences of polynomials generated by the recurrence relation.

2. Let $\left\{f_{n}\right\}$ be any sequence of complex numbers satisfying a three-term recurrence relation

$$
f_{n+1}=\beta_{n} f_{n}-\alpha_{n} f_{n-1}, \quad n \geq 0, f_{0}=1, f_{-1}=0 .
$$

We can associate to $\left\{f_{n}\right\}$ the infinite tridiagonal matrix

$$
D=\left(\begin{array}{cccc}
\beta_{0} & \alpha_{1} & 0 & \ldots \\
1 & \beta_{1} & \alpha_{2} & \ldots \\
0 & 1 & \beta_{2} & \ldots \\
\vdots & \vdots & \vdots & \ddots
\end{array}\right)
$$

As above, by $D^{(m)}$ we denote the infinite tri-diagonal matrix obtained after eliminating from $D$ the first $m$ rows and $m$ columns $\left(D^{(0)}=D\right) . D_{n}^{(m)}$ denotes the principal section of order $n$ of $D^{(m)}$. Define

$$
\Delta_{n}^{(m)}=\operatorname{det} D_{n}^{(m)}, \Delta_{0}^{(m)} \equiv 1
$$


As in Lemma 3 one can prove that

$$
f_{n}=\Delta_{n}^{(0)}, \quad n \geq 0
$$

The relations stated in the next Lemma follow by induction and consecutive use of the three-term recurrence relation. They are well known for the case of orthogonal polynomials on the real line and may be proved in this general situation exactly the same way, so we simply refer to Lemma 5 in [2] (see also [6] and [24]).

Lemma 4. Let $n \in \mathbb{Z}_{+}$and $s \in \mathbb{N}$. Then

$$
0=\Delta_{s-1}^{(n+1)} f_{n+s+1}-\Delta_{s}^{(n+1)} f_{n+s}+\left(\alpha_{n+1} \cdots \alpha_{n+s}\right) f_{n}
$$

and

$$
0=f_{n+s+1}-\Delta_{s}^{(n+1)} f_{n+1}+\alpha_{n+1} \Delta_{s-1}^{(n+2)} f_{n} .
$$

Finally, for $s \in \mathbb{N}, s \geq 2$ and $n \geq s$

$$
\begin{gathered}
0=\Delta_{s-1}^{(n-s+1)} f_{n+s}-\left(\Delta_{s-1}^{(n+1)} \Delta_{s}^{(n-s+1)}-\alpha_{n+1} \Delta_{s-2}^{(n+2)} \Delta_{s-1}^{(n-s+1)}\right) f_{n} \\
+\left(\alpha_{n-s+1} \cdots \alpha_{n}\right) \Delta_{s-1}^{(n+1)} f_{n-s} .
\end{gathered}
$$

Let us assume that the sequences $\left\{\alpha_{n}\right\}$ and $\left\{\beta_{n}\right\}$ have period $k$, then

$$
\Delta_{k}^{(n+1)}=\Delta_{k}^{(n-k+1)}, \Delta_{k-1}^{(n+1)}=\Delta_{k-1}^{(n-k+1)} .
$$

If $\Delta_{k-1}^{(n+1)} \neq 0$ for all $n$, then from (22) we see that the sequence $\left\{f_{n}\right\}$ also satisfies the following three-term recurrence relation over the period $k$ :

$$
0=f_{n+k}-\left(\Delta_{k}^{(n+1)}-\alpha_{n+1} \Delta_{k-2}^{(n+2)}\right) f_{n}+\left(\alpha_{n-k+1} \cdots \alpha_{n}\right) f_{n-k}, \quad n \geq k .
$$

(In case of the polynomials $\Delta_{k-1}^{(n+1)}(z)$, the assumption of being different from zero is unnecessary because (23) would hold except on a finite number of points $z$, thus it would hold for all z.) Because of the periodicity, the factor $\left(\alpha_{n-k+1} \cdots \alpha_{n}\right)$ does not depend on $n$. The next Lemma shows that the other coefficient is also independent of $n$. For a proof we refer to Lemma 2 in [2] (see also [6] and [24]). Again it is an easy consequence of a three-term recurrence relation. In this case

$$
\Delta_{s}^{(m)}=\beta_{m} \Delta_{s-1}^{(m+1)}-\alpha_{m+1} \Delta_{s-2}^{(m+2)}, s \geq 1, m \geq 0,
$$

which may be obtained expanding $\Delta_{s}^{(m)}$ along its first row $\left(\Delta_{-1}^{(m)} \equiv 0\right)$.

Lemma 5. If the sequences $\left\{\alpha_{n}\right\}$ and $\left\{\beta_{n}\right\}$ have period $k$ (and $\Delta_{k-1}^{(n)} \neq 0$ for all $n$ ), then

$$
\Delta_{k}^{(n)}-\alpha_{n} \Delta_{k-2}^{(n+1)}=\Delta_{k}^{(1)}-\alpha_{1} \Delta_{k-2}^{(2)}, n \geq 0
$$

and

$$
0=f_{n+k}-\left(\Delta_{k}^{(1)}-\alpha_{1} \Delta_{k-2}^{(2)}\right) f_{n}+\left(\alpha_{1} \cdots \alpha_{k}\right) f_{n-k}, \quad n \geq k
$$


We call attention to the fact that formulas (23) and (25) may easily be extended to the case when periodicity takes place from a certain index on. This is so because our matrices $D_{m}^{(n)}$, whose determinant form our coefficients, "forget" what occurred before the index $n$ appeared. In fact, that is why we chose $\Delta_{k}^{(1)}-\alpha_{1} \Delta_{k-2}^{(2)}$ as coefficient in (25) (instead of $\left.\Delta_{k}^{(0)}-\alpha_{0} \Delta_{k-2}^{(1)}\right)$ since in our application of Lemma 5 below periodicity occurs for $n \geq 1$ (not for $n \geq 0$ ).

3. Now, we choose a convenient representative in each class $M_{\Gamma}\left(a_{1}, \ldots, a_{k} ; b_{1}, \ldots, b_{k}\right)$. This class is defined in terms of the parameters $\left\{a_{1}, \ldots, a_{k} ; b_{1}, \ldots, b_{k}\right\}$. From (5) it is obvious that in order to have some measure in $M_{\Gamma}\left(a_{1}, \ldots, a_{k} ; b_{1}, \ldots, b_{k}\right)$ the parameters $\left\{a_{1}, \ldots, a_{k}\right\}$ and $\left\{b_{1}, \ldots, b_{k}\right\}$ must satisfy some linking relations. We only consider cases when $M_{\Gamma}\left(a_{1}, \ldots, a_{k} ; b_{1}, \ldots, b_{k}\right) \neq \emptyset$. That is when it contains at least one measure.

Lemma 6. Assume that $a_{j} \in(0,1), j=1, \ldots, k$. Then, there exists

$$
\bar{\mu} \in M_{\Gamma}\left(a_{1}, \ldots, a_{k} ; b_{1}, \ldots, b_{k}\right)
$$

whose sequence of reflection coefficients $\left\{\Psi_{n}(0)\right\}$ satisfies

$$
\Psi_{0}(0)=1, \Psi_{n}(0)=a_{j} e^{i\left(\theta_{1}+\cdots+\theta_{n}\right)}, n=j \bmod k, n \in \mathbb{N},
$$

where $\theta_{j}=\arg b_{j}$ for $j=1, \ldots, k$, and $\theta_{n+k}=\theta_{n}$, for all positive integers $n$.

Proof. Since $M_{\Gamma}\left(a_{1}, \ldots, a_{k} ; b_{1}, \ldots, b_{k}\right) \neq \emptyset$ and $\min \left\{a_{1}, \ldots, a_{k}\right\}>0$, we have (see (5)) that

$$
\left|b_{j}\right|=\frac{a_{j}}{a_{j-1}}, j=1, \ldots, k, \quad\left(a_{0}=a_{k}\right) .
$$

Taking the sequence $\left\{\Psi_{n}(0)\right\}$ as defined above, obviously it constitutes a sequence of reflection coefficients because $\max \left\{a_{1}, \ldots, a_{k}\right\}<1$. Let $\bar{\mu}$ denote the corresponding measure. It rests to show that it belongs to the desired class. In fact, from (26) and (27), we have

$$
\left|\Psi_{n}(0)\right|=a_{j}, \frac{\Psi_{n}(0)}{\Psi_{n-1}(0)}=\frac{a_{j}}{a_{j-1}} e^{i \theta_{n}}=\left|b_{j}\right| e^{i \arg b_{j}}=b_{j}, n=j \bmod k, n \geq 2,
$$

from which (5) immediately follows.

We are interested in finding the support of $\bar{\mu}$. To this end, we will establish a connection between $\bar{\mu}$ and another probability measure $\widehat{\mu}$ whose reflection coefficients are periodic. The support of measures with periodic reflection coefficients has been studied by Ya. L. Geronimus [7] and recently by F. Peherstorfer and R. Steinbauer (see [17]-[20]). We make use of their results and some general ones from operator theory to describe the support of those measures in the classes we have introduced.

Lemma 7. Under the assumptions and notations of Lemma 6, set

$$
A_{j}=a_{j} e^{i\left[\left(\theta_{1}+\cdots+\theta_{j}\right)-j \theta\right]}, j=1, \ldots, k,
$$

where $\theta=\frac{\theta_{1}+\cdots+\theta_{k}}{k}$. Then, there exists a probability measure $\widehat{\mu}$ whose sequence of reflection coefficients $\left\{\widehat{\Psi}_{n}(0)\right\}$ satisfy

$$
\widehat{\Psi}_{n}(0)=A_{j}, n=j \bmod k, j=1, \ldots, k, n \in \mathbb{N} .
$$


For each $n \in \mathbb{Z}_{+}$, we have

$$
\widehat{\Psi}_{n}(z)=e^{-i n \theta} \Psi_{n}\left(e^{i \theta} z\right)
$$

and

$$
\operatorname{supp}(\bar{\mu})=\left\{z \in \Gamma: z e^{-i \theta} \in \operatorname{supp}(\widehat{\mu})\right\}
$$

Proof. Let $\bar{\gamma}=\operatorname{supp}(\bar{\mu})$ and consider the change of variables $z=e^{i \theta} \zeta$. Then

$$
0=\int_{\bar{\gamma}} z^{-\nu} \Psi_{n}(z) d \bar{\mu}(z)=e^{-i \nu \theta} \int_{\widehat{\gamma}} \zeta^{-\nu} \Psi_{n}\left(e^{i \theta} \zeta\right) d \bar{\mu}\left(e^{i \theta} \zeta\right)
$$

where

$$
\widehat{\gamma}=\left\{\zeta=e^{-i \theta} z: z \in \bar{\gamma}\right\}
$$

is the support of $d \widehat{\mu}(\zeta)=d \bar{\mu}\left(e^{i \theta} \zeta\right)$. Therefore,

$$
\widehat{\Psi}_{n}(\zeta)=e^{-i n \theta} \Psi_{n}\left(e^{i \theta} \zeta\right)
$$

is the n-th monic orthogonal polynomial with respect to $\widehat{\mu}$. The reflection coefficients are equal to

$$
\widehat{\Psi}_{n}(0)=e^{-i n \theta} \Psi_{n}(0)=a_{j} e^{i\left[\left(\theta_{1}+\cdots+\theta_{n}\right)-n \theta\right]}=A_{j}, n=j \bmod k, n \in \mathbb{N} .
$$

Thus we have proved (28)-(29), while (30) follows from (31).

4. In the sequel, we will denote by $\tau_{n}$ the leading coefficient of the $\mathrm{n}$-th orthonormal polynomial $\psi_{n}$ with respect to $\bar{\mu}\left(\psi_{n}=\tau_{n} \Psi_{n}\right)$. Using the theory of finite differences, we can obtain a compact expression (and the asymptotic behavior) for the monic orthogonal polynomials $\Psi_{n}(z)$ with respect to the measure $\bar{\mu}$. This is done in terms of the algebraic functions which represent the roots of the characteristic equation

$$
0=\lambda^{2}-p(z) \lambda+z^{k} \frac{\Psi_{k+1}(0)}{\Psi_{1}(0) \tau_{k}^{2}}
$$

where

$$
p(z)=\Delta_{k}^{(1)}(z)-z \frac{\Psi_{2}(0)}{\Psi_{1}(0) \tau_{1}^{2}} \Delta_{k-2}^{(2)}(z),
$$

and the determinants $\Delta_{k}^{(1)}(z), \Delta_{k-2}^{(2)}(z)$ are constructed according to (17)-(18) for the sequence $\left\{\Psi_{n}(0)\right\}$ of reflection coefficients relative to $\bar{\mu}$.

Denote

$$
G_{ \pm}(z)=\frac{1}{2}\left(p(z) \pm \sqrt{p^{2}(z)-4 z^{k} \frac{\Psi_{k+1}(0)}{\Psi_{1}(0) \tau_{k}^{2}}}\right)
$$

the two algebraic functions which give the roots of (32). They are defined analytically on the complement of the set

$$
\gamma(\bar{\mu})=\left\{z:-1 \leq \frac{\tau_{k} p(z) \Psi_{1}(0)^{1 / 2}}{2 z^{k / 2} \Psi_{k+1}(0)^{1 / 2}} \leq 1\right\}
$$

which is the set of points for which both roots coincide in modulus. In (34), we take, for example, the root which is positive for $z=x>0$. The set $\gamma(\bar{\mu})$ consists of at most $k$ 
compact analytic curves, each one contained in one of the at most $k$ connected components of the closed region

$$
\left\{z:\left|\frac{\tau_{k} p(z) \Psi_{1}(0)^{1 / 2}}{2 z^{k / 2} \Psi_{k+1}(0)^{1 / 2}}\right|^{2} \leq 1\right\} .
$$

(By the minimum principle each such component must contain at least one zero of $p^{2}(z)$ and this polynomial has at most $k$ distinct zeros.) In (33), we define $G_{+}(z)$ to be the root of greater modulus on $\mathbb{C} \backslash \gamma(\bar{\mu})$. On $\gamma(\bar{\mu})$ we define $G_{+}(z)$ (and $G_{-}(z)$ ) by continuity considering the arc $\gamma(\bar{\mu})$ to have two sides as it is usually done.

The next Lemma is a reformulation of Theorem 2.1 in [19] in terms more appropriate for our further considerations. Recall that $\psi_{n}=\tau_{n} \Psi_{n}$ denotes the n-th orthonormal polynomial with respect to $\bar{\mu}$ and $\tau_{n}>0$ its leading coefficient. In the sequel, $(\cdot)^{\prime}$ denotes the derived set of $(\cdot)$.

Lemma 8. Assume that $a_{j} \in(0,1), j=1, \ldots, k$. Let $\bar{\mu}$ be the measure constructed in Lemma 6 and $\left\{\psi_{n}\right\}$ the corresponding sequence of orthonormal polynomials. We have

$$
\Psi_{n}(z)=\frac{\Psi_{k+j}(z)-G_{-}(z) \Psi_{j}(z)}{G_{+}(z)-G_{-}(z)} G_{+}^{\nu}(z)+\frac{G_{+}(z) \Psi_{j}(z)-\Psi_{k+j}(z)}{G_{+}(z)-G_{-}(z)} G_{-}^{\nu}(z)
$$

for $n=k \nu+j, \nu \geq 2, j=1, \ldots, k$, whenever $G_{+}(z) \neq G_{-}(z)$. In particular,

$$
\lim _{\nu}\left[\psi_{n}(z)-\frac{\Psi_{k+j}(z)-G_{-}(z) \Psi_{j}(z)}{G_{+}(z)-G_{-}(z)}\left(\tau_{k} G_{+}(z)\right)^{\nu} \tau_{j}\right]=0
$$

uniformly on each compact subset of $\mathbb{C} \backslash \gamma(\bar{\mu})$. Moreover,

$$
\gamma(\bar{\mu})=(\operatorname{supp}(\bar{\mu}))^{\prime}
$$

and

$$
\bigcap_{j=1}^{k}\left\{z: \Psi_{k+j}(z)=G_{-}(z) \Psi_{j}(z)\right\} \backslash \gamma(\bar{\mu})=\operatorname{supp}(\bar{\mu}) \backslash \gamma(\bar{\mu})
$$

consists of at most a finite number of points.

\section{Proof.}

Notice that for the sequence $\left\{\Psi_{n}(0)\right\}$, we have:

$$
z+\frac{\Psi_{n+1}(0)}{\Psi_{n}(0)}=z+b_{j+1}=z+\frac{\Psi_{j+1}(0)}{\Psi_{j}(0)},
$$

and

$$
z \frac{\Psi_{n+1}(0)}{\Psi_{n}(0)}\left(\frac{\tau_{n-1}}{\tau_{n}}\right)^{2}=z b_{j+1}\left(1-a_{j}^{2}\right)=z \frac{\Psi_{j+1}(0)}{\Psi_{j}(0)}\left(\frac{\tau_{j-1}}{\tau_{j}}\right)^{2},
$$

where $n=j \bmod k, n \geq 1, j=1, \ldots, k\left(b_{k+1}=b_{1}\right)$. Therefore, the corresponding matrix (17) is $k$ periodic if we disregard the first row and column. Thus, from Lemma 5 (see the observation made just after that Lemma), we obtain

$$
\Psi_{n+k}(z)=p(z) \Psi_{n}(z)-z^{k} \frac{\Psi_{k+1}(0)}{\Psi_{1}(0) \tau_{k}^{2}} \Psi_{n-k}(z)
$$


Consider the trivial equation

$$
\Psi_{n}(z)=\Psi_{n}(z)
$$

In matrix form, the system (39)-(40) can be written

$$
\left(\begin{array}{c}
\Psi_{n+k}(z) \\
\Psi_{n}(z)
\end{array}\right)=M(z)\left(\begin{array}{c}
\Psi_{n}(z) \\
\Psi_{n-k}(z)
\end{array}\right)
$$

where

$$
M(z)=\left(\begin{array}{cc}
p(z) & -z^{k} \frac{\Psi_{k+1}(0)}{\Psi_{1}(0) \tau_{k}^{2}} \\
1 & 0
\end{array}\right) .
$$

The eigenvalues of $M(z)$ are the roots $G_{ \pm}(z)$ of the characteristic equation (32). The corresponding eigenvectors (which form a basis if $G_{+}(z) \neq G_{-}(z)$ ) are

$$
\left(\begin{array}{c}
G_{+}(z) \\
1
\end{array}\right),\left(\begin{array}{c}
G_{-}(z) \\
1
\end{array}\right) \text {. }
$$

Take $n=\nu k+j, j=1, \ldots, k$ fixed. It is easily verified that

$$
\left(\begin{array}{c}
\Psi_{j+k}(z) \\
\Psi_{j}(z)
\end{array}\right)=c_{1}(z)\left(\begin{array}{c}
G_{+}(z) \\
1
\end{array}\right)+c_{2}(z)\left(\begin{array}{c}
G_{-}(z) \\
1
\end{array}\right)
$$

where

$$
c_{1}(z)=\frac{\Psi_{k+j}(z)-G_{-}(z) \Psi_{j}(z)}{G_{+}(z)-G_{-}(z)}, c_{2}(z)=\frac{G_{+}(z) \Psi_{j}(z)-\Psi_{k+j}(z)}{G_{+}(z)-G_{-}(z)} .
$$

Therefore, we have (35) since

$$
\begin{gathered}
\left(\begin{array}{c}
\Psi_{n+k}(z) \\
\Psi_{n}(z)
\end{array}\right)=M^{\nu}(z)\left(\begin{array}{c}
\Psi_{j+k}(z) \\
\Psi_{j}(z)
\end{array}\right)= \\
=c_{1}(z) G_{+}^{\nu}(z)\left(\begin{array}{c}
G_{+}(z) \\
1
\end{array}\right)+c_{2}(z) G_{-}^{\nu}(z)\left(\begin{array}{c}
G_{-}(z) \\
1
\end{array}\right) .
\end{gathered}
$$

Now, we obtain a weaker version of (36). In $\mathbb{C} \backslash \gamma(\bar{\mu})$, we have $\left|\frac{G_{-}(z)}{G_{+}(z)}\right|<1$. Thus, its supremum is strictly less than 1 on any compact subset of $\mathbb{C} \backslash \gamma(\bar{\mu})$. Using (35), we get

$$
\lim _{\nu} \frac{\Psi_{n}(z)}{G_{+}^{\nu}(z)}=\frac{\Psi_{k+j}(z)-G_{-}(z) \Psi_{j}(z)}{G_{+}(z)-G_{-}(z)},
$$

uniformly on each compact subset of $\mathbb{C} \backslash \gamma(\bar{\mu})$. Moreover,

$$
\lim _{\nu} \frac{\Psi_{n}(z)}{G_{-}^{\nu}(z)}=\frac{G_{+}(z) \Psi_{j}(z)-\Psi_{k+j}(z)}{G_{+}(z)-G_{-}(z)}
$$

for $z \in\left\{z: \Psi_{k+j}(z)=G_{-}(z) \Psi_{j}(z)\right\} \backslash \gamma(\bar{\mu})$.

Let us see that the set $\left\{z: \Psi_{k+j}(z)=G_{-}(z) \Psi_{j}(z)\right\} \backslash \gamma(\bar{\mu})$ contains at most a finite number of points. In fact, at any such point, taking $\lambda=G_{-}(z)$ in (32), we obtain

$$
0=\Psi_{k+j}^{2}(z)-p(z) \Psi_{k+j}(z) \Psi_{j}(z)+z^{k} \frac{\Psi_{k+1}(0)}{\Psi_{1}(0) \tau_{k}^{2}} \Psi_{j}^{2}(z)
$$


This equation is either solved identically or it has at most $2 k+2 j$ zeros. If (43) is solved identically, then

$$
G_{+}(z) \equiv \frac{\Psi_{k+j}(z)}{\Psi_{j}(z)}, z \in \mathbb{C},
$$

because $G_{+}(z)$ is the only one of the functions which are roots of (32) that tends to infinity when $z$ tends to infinity. Then, any point for which $\Psi_{k+j}(z)=G_{-}(z) \Psi_{j}(z)$ would have to be in $\gamma(\bar{\mu})$. In the second case, we have nothing else to prove.

From (41) and the assertion just proved above, using Hurwtiz' Theorem, we obtain that on any fixed compact subset $K \subset \mathbb{C} \backslash\left(\gamma(\bar{\mu}) \cup\left\{z: \Psi_{k+j}(z)=G_{-}(z) \Psi_{j}(z)\right\}\right), \Psi_{n}(z) \neq$ $0, z \in K, n=\nu k+j$, for all sufficiently large $\nu$. Therefore, using (41)

$$
\lim _{\nu} \frac{\Psi_{n+k}(z)}{\Psi_{n}(z)}=G_{+}(z)
$$

uniformly on each compact subset of $\mathbb{C} \backslash\left(\gamma(\bar{\mu}) \cup\left\{z: \Psi_{k+j}(z)=G_{-}(z) \Psi_{j}(z)\right\}\right)$. On $\{z$ : $\left.\Psi_{k+j}(z)=G_{-}(z) \Psi_{j}(z)\right\} \backslash \gamma(\bar{\mu})$, we have that

$$
\lim _{\nu} \frac{\Psi_{n+k}(z)}{\Psi_{n}(z)}=G_{-}(z)
$$

Since

$$
\tau_{n}=\tau_{k}^{\nu} \tau_{j}
$$

then (44) and (45) give

$$
\lim _{\nu} \frac{\psi_{n+k}(z)}{\psi_{n}(z)}=\tau_{k} G_{+}(z)
$$

uniformly on each compact subset of $\mathbb{C} \backslash\left(\gamma(\bar{\mu}) \cup\left\{z: \Psi_{k+j}(z)=G_{-}(z) \Psi_{j}(z)\right\}\right)$, and

$$
\lim _{\nu} \frac{\psi_{n+k}(z)}{\psi_{n}(z)}=\tau_{k} G_{-}(z)
$$

on $\left\{z: \Psi_{k+j}(z)=G_{-}(z) \Psi_{j}(z)\right\} \backslash \gamma(\bar{\mu})$.

In order to complete the proof of (36), we need some additional information on the functions $G_{+}$and $G_{-}$which we will extract from the case of periodic reflection coefficients studied in detail in [19].

Denote $\widehat{G}_{+}$and $\widehat{G}_{-}$the functions that appear on the right of (46) and (47) respectively when instead of the sequence $\left\{\Psi_{n}(0)\right\}$ of reflections we consider the associated sequence $\left\{\widehat{\Psi}_{n}(0)\right\}$ of periodic reflection coefficients constructed in Lemma 7 . The $L_{2}$ norms of the orthogonal polynomials $\Psi_{n}$ and $\widehat{\Psi}_{n}$ are equal. Thus, from (29), we have

$$
\frac{\widehat{\psi}_{n+k}(z)}{\widehat{\psi}_{n}(z)}=e^{-i k \theta} \frac{\psi_{n+k}\left(e^{i \theta} z\right)}{\psi_{n}\left(e^{i \theta} z\right)} .
$$

Using (30) and (46), it follows that

$$
\widehat{G}_{+}(z)=e^{-i k \theta} G_{+}\left(e^{i \theta} z\right), \widehat{G}_{-}(z)=e^{-i k \theta} G_{-}\left(e^{i \theta} z\right) .
$$

For the periodic case, the limit (46) may also be derived from Theorem 2.1 (a) in [19]. This allows us to identify our expression for $2 \widehat{G}_{+}(z)$ with what the authors of that 
paper denote $T_{N}(z)+\sqrt{R(z)} U_{N-l}(z)$ and, therefore, $2 \widehat{G}_{-}(z)$ is what they denote $T_{N}(z)-$ $\sqrt{R(z)} U_{N-1}(z)$.

From Lemma 2.1 in [19], (30), and (48), it follows that

$$
\left|\tau_{k} G_{-}(z)\right|<1,\left|\tau_{k} G_{+}(z)\right|>1,
$$

on $\mathbb{C} \backslash(\operatorname{supp}(\bar{\mu}))^{\prime}$ and

$$
\left|\tau_{k} G_{-}(z)\right|=\left|\tau_{k} G_{+}(z)\right|=1,
$$

on $(\operatorname{supp}(\bar{\mu}))^{\prime}$ (see also (2.5) and (2.6) in [19]). From this and the definition of $\gamma(\bar{\mu})$, we obtain (36) and (37). Using Lemma 1, (47), and (49), we obtain (38).

To end this section we offer the following Lemma which states that except for at most a numerable set of (isolated mass) points all the measures in $M_{\Gamma}\left(a_{1}, \ldots, a_{k} ; b_{1}, \ldots, b_{k}\right)$ have the same support, consisting of at most $k$ disjoint arcs contained in $\Gamma$.

Lemma 9. Under the assumptions of Lemma 6, we have that

$$
(\operatorname{supp}(\mu))^{\prime}=\gamma(\bar{\mu})=(\operatorname{supp}(\bar{\mu}))^{\prime} .
$$

Proof. It is not hard to prove that the operator $U(\mu)-U(\bar{\mu})$ is compact (one can use exactly the same arguments as those employed in the case considered for the proof of Theorem 3 in [12]). Thus, from Weyl's Theorem (see [14] and more precisely Problem 182 in [13]), we know that except for isolated points (which are eigenvalues) the spectra of $U(\mu)$ and $U(\bar{\mu})$ coincide. This together with Lemmas 1 and 8 give us the statement.

\section{Ratio Asymptotics}

1. In the sequel, $\Delta_{n}^{(m)}(z)$ is the determinant defined in (18) relative to the sequence $\left\{\Psi_{n}(0)\right\}$ of reflection coefficients of $\bar{\mu}$ while $\delta_{n}^{(m)}(z)$ is that relative to the sequence $\left\{\Phi_{n}(0)\right\}$ of reflection coefficients of $\mu$. We also distinguish $\kappa_{n}$ the leading coefficient of the n-th orthonormal polynomial $\phi_{n}$ with respect to the measure $\mu$ from $\tau_{n}$ the leading coefficient of the n-th orthonormal polynomial $\psi_{n}$ with respect to $\bar{\mu}$. We are ready for the proof of

Theorem 2. Let $\mu \in M_{\Gamma}\left(a_{1}, \ldots, a_{k} ; b_{1}, \ldots, b_{k}\right), a_{j} \in(0,1), j=1, \ldots, k$. Then,

$$
\lim _{n \longrightarrow \infty} \frac{\Phi_{n+k}(z)}{\Phi_{n}(z)}=G_{+}(z), \lim _{n=j \bmod k} \frac{\kappa_{n-1}}{\kappa_{n}}=\sqrt{1-a_{j}^{2}} \in(0,1), j=1, \ldots, k
$$

uniformly on each compact subset of $\mathbb{C} \backslash\left(\bigcup_{s=1}^{k}\left\{z: \Delta_{k-1}^{(s)}(z)=0\right\} \cup \operatorname{supp}(\mu)\right)$. Moreover,

i) $(\operatorname{supp}(\mu))^{\prime}=\gamma(\bar{\mu})$

ii) for any compact $K \subset \mathbb{C} \backslash\left(\bigcup_{s=1}^{k}\left\{z: \Delta_{k-1}^{(s)}(z)=0\right\} \cup \operatorname{supp}(\mu)\right)$, and for all sufficiently large $n$, the polynomials $\Phi_{n}(z)$ have no zeros on $K$. 
Under stronger conditions on the measure $\mu$, some of the statements of Theorem 2 were obtained before by other authors. In connection with $i$ ) see $[8$, Th. IV] and [19, Th. 4.1]. In relation with $i i)$ see [19, Th. 3.3].

The following result complements Theorem 2 .

Theorem 3. Let $z \in \mathbb{C} \backslash\left(\bigcup_{s=1}^{k}\left\{z: \Delta_{k-1}^{(s)}(z)=0\right\} \cup \gamma(\bar{\mu})\right)$. The following conditions are equivalent

i) $z$ is an isolated mass point of $\operatorname{supp}(\mu)$ (isolated eigenvalue of $\sigma(U)$ ),

ii) there exists $j \in\{1,2, \ldots, k\}$ such that

$$
\lim _{\nu} \frac{\Phi_{n+k}(z)}{\Phi_{n}(z)}=G_{-}(z), n=\nu k+j
$$

iii) we have

$$
\lim _{n} \frac{\Phi_{n+k}(z)}{\Phi_{n}(z)}=G_{-}(z)
$$

Other conditions for the existence of isolated mass points may be found in [9, Th. X] and [19, Remark 3.2].

Proof (of Theorems 2 and 3 ).

Let $\mu \in M_{\Gamma}\left(a_{1}, \ldots, a_{k} ; b_{1}, \ldots, b_{k}\right)$. Then $M_{\Gamma}\left(a_{1}, \ldots, a_{k} ; b_{1}, \ldots, b_{k}\right) \neq \emptyset$ and we may construct $\bar{\mu}$ by Lemma 6 . From (22), we have that

$$
0=\Phi_{n+k}(z)-\alpha_{n, 1}(z) \Phi_{n}(z)+\alpha_{n, 2}(z) \Phi_{n-k}(z), n \geq k,
$$

whenever $\delta_{k-1}^{(n-k+1)}(z) \neq 0$, where

$$
\alpha_{n, 1}(z)=\frac{\delta_{k-1}^{(n+1)}(z) \delta_{k}^{(n-k+1)}(z)}{\delta_{k-1}^{(n-k+1)}(z)}-z \frac{\Phi_{n+2}(0)}{\Phi_{n+1}(0)}\left(\frac{\kappa_{n}}{\kappa_{n+1}}\right)^{2} \delta_{k-2}^{(n+2)}(z)
$$

and

$$
\alpha_{n, 2}(z)=z^{k} \frac{\Phi_{n+1}(0)}{\Phi_{n-k+1}(0)}\left(\frac{\kappa_{n-k}}{\kappa_{n}}\right)^{2} \frac{\delta_{k-1}^{(n+1)}(z)}{\delta_{k-1}^{(n-k+1)}(z)} .
$$

Because of (5), (8) and Lemma 5, we have that

$$
\begin{gathered}
\lim _{n=j \bmod k} \delta_{m}^{(n)}(z)=\Delta_{m}^{(j)}(z), \\
\lim _{n \longrightarrow \infty} \alpha_{n, 1}(z)=p(z)
\end{gathered}
$$

and

$$
\lim _{n \longrightarrow \infty} \alpha_{n, 2}(z)=z^{k} \frac{\Psi_{k+1}(0)}{\Psi_{1}(0) \tau_{k}^{2}}
$$

These limits are uniform on any compact subset of $\mathbb{C} \backslash\left(\bigcup_{s=1}^{k}\left\{z: \Delta_{k-1}^{(s)}(z)=0\right\}\right)$, because the degrees of the polynomials involved remain bounded from above. 
Fix $j=1, \ldots, k$ and $z_{0} \in \Omega=\mathbb{C} \backslash\left(\bigcup_{s=1}^{k}\left\{z: \Delta_{k-1}^{(s)}(z)=0\right\} \cup \gamma(\bar{\mu})\right)$. Take a closed neighborhood $B$ of $z_{0}$ also contained in $\Omega$. Because of (54) (for $m=k-1$ ), there exists $\nu_{0}=\nu_{0}\left(z_{0}\right)$, such that for all $z \in B$ and $\nu \geq \nu_{0}$, we have that $\delta_{k-1}^{n-k+1}(z) \neq 0$, and the sequence $\left\{\Phi_{n}(z)\right\}, n=\nu k+j, \nu \in \mathbb{N}$, satisfies the three-term recurrence relation (53) for all $z \in B$ and $\nu \geq \nu_{0}$. In the sequel, given $z_{0} \in \Omega$, we only consider such values of $\nu$ and $n$. Notice that if for some $z \in B, \Phi_{n}(z)=\Phi_{n+k}(z)=0$ for a given $n$ then $\Phi_{n}(z)=0$ for all $n=\nu k+j, \nu \geq \nu_{0}-1$.

According to (55) and (56) the coefficients in the three-term recurrence relation have limits (independent of $j$ ) and they are the coefficients of the characteristic equation (32). Since $z_{0} \in \mathbb{C} \backslash \gamma(\bar{\mu})$, the roots of the characteristic equation $G_{ \pm}\left(z_{0}\right)$ are different in modulus. Because of all this, we may use a Theorem of H. Poincaré (see [5, Ch. V, §5, pp 327]) which states that under these conditions, either $\Psi_{n}\left(z_{0}\right)=0$ for all $\nu \geq \nu_{0}-1$ or there exists

$$
\lim _{\nu} \frac{\Phi_{n+k}\left(z_{0}\right)}{\Phi_{n}\left(z_{0}\right)}, n=\nu k+j,
$$

being this limit one of two roots of the characteristic equation; that is, either $G_{+}\left(z_{0}\right)$ or $G_{-}\left(z_{0}\right)$.

Let us prove that the limit always exists even in the case when $\Psi_{n}\left(z_{0}\right)=0, \nu \geq \nu_{0}-1$. In fact, if this is the case, we have that $z_{0}$ is a common zero for all the polynomials $\Psi_{n}, \nu \geq \nu_{0}-1$. Let $h$ be the largest integer such that $\left(z-z_{0}\right)^{h}$ divides all $\Psi_{n}, \nu \geq \nu_{0}-1$. Since $\operatorname{deg} \Psi_{n}=n$ for all $n$, we have that $h<\infty$. Take $\widehat{\Psi}_{n}(z)=\Psi_{n}(z) /\left(z-z_{0}\right)^{h}$. The sequence $\left\{\widehat{\Psi}_{n}\right\}$ also satisfies the three term recurrence relation (53) for all $z \in B$ and $\nu \geq \nu_{0}$ and $\widehat{\Psi}_{n}\left(z_{0}\right) \neq 0$ for at least one $\nu \geq \nu_{0}$. Therefore, by Poincare's Theorem there exists

$$
\lim _{\nu} \frac{\widehat{\Psi}_{n+k}\left(z_{0}\right)}{\widehat{\Psi}_{n}\left(z_{0}\right)}=\lim _{\nu} \frac{\Psi_{n+k}\left(z_{0}\right)}{\Psi_{n}\left(z_{0}\right)}
$$

because the new sequence of polynomials evaluated at $z_{0}$ does not give the trivial solution of the difference equation. (That is, the second limit above must be understood in the sense of the values obtained in the ratio after cancelling out common factors.)

We must determine which one of these values is the limit for a given $z \in \Omega$. Before doing this, we will prove that if for certain $j=1, \ldots, k$ and $z \in \Omega$ the limit is $G_{-}(z)$ then for all $j=1, \ldots, k$ the limit is $G_{-}(z)$. Once this is done, it is obvious that the analogous statement is true if the limit is $G_{+}(z)$. Certainly, if $k=1$ there is nothing to be proved. So we consider that $k \geq 2$.

Assume that

$$
\lim _{\nu} \frac{\Phi_{n+k}(z)}{\Phi_{n}(z)}=G_{-}(z), n=\nu k+j,
$$

for given $j \in\{1, \ldots, k\}$ and $z \in \Omega$. From (20), we have that

$$
0=\delta_{k-1}^{(n+1)}(z) \frac{\Phi_{n+k+1}(z)}{\Phi_{n+k}(z)}-\delta_{k}^{(n+1)}(z)+z^{k} \frac{\Phi_{n+k+1}(0)}{\Phi_{n+1}(0)}\left(\frac{\kappa_{n}}{\kappa_{n+k}}\right)^{2} \frac{\Phi_{n}(z)}{\Phi_{n+k}(z)} .
$$

Using (54), (5) and (57), we obtain $(n=\nu k+j)$

$$
\lim _{\nu} \frac{\Phi_{n+k+1}(z)}{\Phi_{n+k}(z)}=\frac{1}{\Delta_{k-1}^{(j+1)}(z)}\left[\Delta_{k}^{(j+1)}(z)-z^{k} \frac{\Psi_{k+1}(0)}{\Psi_{1}(0)}\left(\frac{1}{\tau_{k}}\right)^{2} \frac{1}{G_{-}(z)}\right]
$$




$$
=\frac{1}{\Delta_{k-1}^{(j+1)}(z)}\left[\Delta_{k}^{(j+1)}(z)-G_{+}(z)\right]
$$

(notice that for $z \in \Omega, \Delta_{k-1}^{(j+1)}(z) \neq 0$ ). In (59) we have also used the fact that

$$
G_{+}(z) G_{-}(z)=z^{k} \frac{\Psi_{k+1}(0)}{\Psi_{1}(0)}\left(\frac{1}{\tau_{k}}\right)^{2} .
$$

If $\Delta_{k}^{(j+1)}(z)-G_{+}(z) \neq 0$, then from $(59)$

$$
\lim _{\nu} \frac{\Phi_{n+k+1}(z)}{\Phi_{n+k}(z)} \frac{\Phi_{n}(z)}{\Phi_{n+1}(z)}=1
$$

and using (57) it follows that

$$
\lim _{\nu} \frac{\Phi_{n+k+1}(z)}{\Phi_{n+1}(z)}=G_{-}(z)
$$

or what is the same, (57) takes place substituting $j$ by $j+1$.

We will now show that $G_{+}(z)=\Delta_{k}^{(j+1)}(z)$ implies that $z \in \bigcup_{s=1}^{k}\left\{z: \Delta_{k-1}^{(s)}(z)=0\right\}$. In fact, $G_{+}(z)=\Delta_{k}^{(j+1)}(z)$ yields that

$$
\begin{gathered}
0=\left(\Delta_{k}^{(j+1)}(z)\right)^{2}-p(z) \Delta_{k}^{(j+1)}(z)+z^{k} \frac{\Psi_{k+1}(0)}{\Psi_{1}(0)}\left(\frac{1}{\tau_{k}}\right)^{2}=\left(\Delta_{k}^{(j+1)}(z)\right)^{2} \\
-\left(\Delta_{k}^{(j+1)}(z)-z \frac{\Psi_{j+2}(0)}{\Psi_{j+1}(0)}\left(\frac{\tau_{j}}{\tau_{j+1}}\right)^{2} \Delta_{k-2}^{(j+2)}(z)\right) \Delta_{k}^{(j+1)}(z)+z^{k} \frac{\Psi_{k+1}(0)}{\Psi_{1}(0)}\left(\frac{1}{\tau_{k}}\right)^{2} \\
=z \frac{\Psi_{j+2}(0)}{\Psi_{j+1}(0)}\left(\frac{\tau_{j}}{\tau_{j+1}}\right)^{2} \Delta_{k}^{(j+1)}(z) \Delta_{k-2}^{(j+2)}(z)+z^{k} \frac{\Psi_{k+1}(0)}{\Psi_{1}(0)}\left(\frac{1}{\tau_{k}}\right)^{2} .
\end{gathered}
$$

On the other hand, we have the identity (valid for all $z \in \mathbb{C}$ )

$$
\Delta_{k-1}^{(j+1)}(z) \Delta_{k-1}^{(j+2)}(z)-\Delta_{k}^{(j+1)}(z) \Delta_{k-2}^{(j+2)}(z)=z^{k-1} \frac{\Psi_{j+k+1}(0)}{\Psi_{j+2}(0)}\left(\frac{\tau_{j+1}}{\tau_{j+k}}\right)^{2} .
$$

To prove this identity notice that (see (24))

$$
\begin{aligned}
& \Delta_{k-1}^{(j+1)}(z)=\left(z+\frac{\Psi_{j+2}(0)}{\Psi_{j+1}(0)}\right) \Delta_{k-2}^{(j+2)}(z)-z \frac{\Psi_{j+3}(0)}{\Psi_{j+2}(0)}\left(\frac{\tau_{j+1}}{\tau_{j+2}}\right)^{2} \Delta_{k-3}^{(j+3)}(z), \\
& \Delta_{k}^{(j+1)}(z)=\left(z+\frac{\Psi_{j+2}(0)}{\Psi_{j+1}(0)}\right) \Delta_{k-1}^{(j+2)}(z)-z \frac{\Psi_{j+3}(0)}{\Psi_{j+2}(0)}\left(\frac{\tau_{j+1}}{\tau_{j+2}}\right)^{2} \Delta_{k-2}^{(j+3)}(z) .
\end{aligned}
$$

Multiplying the first equality by $\Delta_{k-1}^{(j+2)}(z)$, the second by $\Delta_{k-2}^{(j+2)}(z)$ and deleting the second from the first, we get

$$
\begin{aligned}
& \Delta_{k-1}^{(j+1)}(z) \Delta_{k-1}^{(j+2)}(z)-\Delta_{k}^{(j+1)}(z) \Delta_{k-2}^{(j+2)}(z)= \\
& =z \frac{\Psi_{j+3}(0)}{\Psi_{j+2}(0)}\left(\frac{\tau_{j+1}}{\tau_{j+2}}\right)^{2}\left(\Delta_{k-2}^{(j+2)}(z) \Delta_{k-2}^{(j+3)}(z)-\Delta_{k-1}^{(j+2)}(z) \Delta_{k-3}^{(j+3)}(z)\right) .
\end{aligned}
$$


Repeating this process with the right-hand side $(k-1)$ times, we obtain

$$
\begin{aligned}
& \Delta_{k-1}^{(j+1)}(z) \Delta_{k-1}^{(j+2)}(z)-\Delta_{k}^{(j+1)}(z) \Delta_{k-2}^{(j+2)}(z)= \\
& =z^{k-1} \frac{\Psi_{j+k+1}(0)}{\Psi_{j+2}(0)}\left(\frac{\tau_{j+1}}{\tau_{j+k}}\right)^{2}\left(\Delta_{0}^{(j+k)}(z) \Delta_{0}^{(j+k+1)}(z)-\Delta_{1}^{(j+k)}(z) \Delta_{-1}^{(j+k+1)}(z)\right) \\
& =z^{k-1} \frac{\Psi_{j+k+1}(0)}{\Psi_{j+2}(0)}\left(\frac{\tau_{j+1}}{\tau_{j+k}}\right)^{2}
\end{aligned}
$$

$\left(\Delta_{0}^{(m)}(z) \equiv 1\right.$ and $\left.\Delta_{-1}^{(m)}(z) \equiv 0\right)$ which is $(61)$.

Multiplying (61) times $z \frac{\Psi_{j+2}(0)}{\Psi_{j+1}(0)}\left(\frac{\tau_{j}}{\tau_{j+1}}\right)^{2}$, we obtain

$$
\begin{gathered}
z \frac{\Psi_{j+2}(0)}{\Psi_{j+1}(0)}\left(\frac{\tau_{j}}{\tau_{j+1}}\right)^{2} \Delta_{k-1}^{(j+1)}(z) \Delta_{k-1}^{(j+2)}(z)-z \frac{\Psi_{j+2}(0)}{\Psi_{j+1}(0)}\left(\frac{\tau_{j}}{\tau_{j+1}}\right)^{2} \Delta_{k}^{(j+1)}(z) \Delta_{k-2}^{(j+2)}(z)= \\
=z^{k} \frac{\Psi_{j+k+1}(0)}{\Psi_{j+1}(0)}\left(\frac{\tau_{j}}{\tau_{j+k}}\right)^{2}=z^{k} \frac{\Psi_{k+1}(0)}{\Psi_{1}(0)}\left(\frac{1}{\tau_{k}}\right)^{2} .
\end{gathered}
$$

If $G_{+}(z)=\Delta_{k}^{(j+1)}(z)$ then (60) takes place and substituting in (62), we obtain

$$
z \frac{\Psi_{j+2}(0)}{\Psi_{j+1}(0)}\left(\frac{\tau_{j}}{\tau_{j+1}}\right)^{2} \Delta_{k-1}^{(j+1)}(z) \Delta_{k-1}^{(j+2)}(z)=0 .
$$

Therefore, either $z=0$ or $z \in \bigcup_{s=1}^{k}\left\{z: \Delta_{k-1}^{(s)}(z)=0\right\}$.

Finally, let us show that at $z=0$

$$
\lim _{n \longrightarrow \infty} \frac{\Phi_{n+k}(0)}{\Phi_{n}(0)}=G_{+}(0)
$$

thus, there is no $j=1, \ldots, k$ such that (57) takes place at $z=0$. Indeed, from (53), we see that

$$
\frac{\Phi_{n+k}(0)}{\Phi_{n}(0)}=\frac{\delta_{k-1}^{(n+1)}(0) \delta_{k}^{(n-k+1)}(0)}{\delta_{k-1}^{(n-k+1)}(0)}
$$

(notice that $\delta_{m}^{(n)}(0) \neq 0$ for all $n$ and $m$ ). Taking limits, we obtain for all $j=1, \ldots, k$ and $n=\nu k+j$

$$
\lim _{\nu} \frac{\Phi_{n+k}(0)}{\Phi_{n}(0)}=\Delta_{k}^{(j+1)}(0)=\frac{\Psi_{j+k+1}(0)}{\Psi_{j+1}(0)}=\frac{\Psi_{k+1}(0)}{\Psi_{1}(0)}=\Delta_{k}^{(1)}(0)=G_{+}(0)
$$

which is what we wanted to prove (here, the second and the last equation follow from (17) and (33), respectively).

Therefore, we have proved that if (57) takes place at $z \in \Omega$ for a given $j$ it is also true for $j+1$. Repeating this procedure for $j+1$ and so on, we obtain the desired result.

Now let us conclude the proof of Theorem 3. From Lemmas 1 and 9, we know that

$$
\operatorname{supp}(\mu) \backslash(\operatorname{supp}(\mu))^{\prime}=\sigma_{p}(U) \backslash \gamma(\bar{\mu}),
$$


that is, isolated mass points of $\mu$ are eigenvalues of $U$ not belonging to $\gamma(\bar{\mu})$. On the other hand, for each $j=1, \ldots, k$ and $z \in \Omega$ we have proved that

$$
\lim _{\nu} \frac{\phi_{n+k}(z)}{\phi_{n}(z)}=\lim _{\nu} \frac{\kappa_{n+k}}{\kappa_{n}} \lim _{\nu} \frac{\Phi_{n+k}(z)}{\Phi_{n}(z)}=\tau_{k} \lim _{\nu} \frac{\Phi_{n+k}(z)}{\Phi_{n}(z)}
$$

where $\lim _{\nu} \frac{\Phi_{n+k}(z)}{\Phi_{n}(z)}$ is either $G_{+}(z)$ or $G_{-}(z)$. Additionally, we know that if the limit is $G_{-}(z)$ for some $j=1, \ldots, k$, then it is $G_{-}(z)$ for all $j=1, \ldots, k$.

Assume that $z \in \Omega$ and for a given $j=1, \ldots, k, \lim _{n=j \bmod k} \frac{\phi_{n+k}(z)}{\phi_{n}(z)}=\tau_{k} G_{-}(z)$. Then

$$
\lim _{n \longrightarrow \infty} \frac{\phi_{n+k}(z)}{\phi_{n}(z)}=\tau_{k} G_{-}(z) .
$$

Since $\left|\tau_{k} G_{-}(z)\right|<1$ (see (49)), it follows that

$$
\left(\phi_{0}(z), \phi_{1}(z), \ldots, \phi_{n}(z), \ldots\right) \in \ell^{2} .
$$

From Lemma 1 and (63), we have that $z \in \operatorname{supp}(\mu) \backslash\left[\bigcup_{s=1}^{k}\left\{z: \Delta_{k-1}^{(s)}(z)=0\right\} \cup(\operatorname{supp}(\mu))^{\prime}\right]$. Therefore, $i i$ ) in Theorem 3 implies $i$ ).

Let $z \in \operatorname{supp}(\mu) \backslash\left[\bigcup_{s=1}^{k}\left\{z: \Delta_{k-1}^{(s)}(z)=0\right\} \cup(\operatorname{supp}(\mu))^{\prime}\right]$. In particular, $z \in \Omega$, therefore for each $j=1, \ldots, k, \lim _{n=j \bmod k} \frac{\Phi_{n+k}(z)}{\Phi_{n}(z)}$ exists. If for some $j=1, \ldots, k$ the limit is $G_{+}(z)$, then $\lim _{n=j \bmod k} \frac{\phi_{n+k}(z)}{\phi_{n}(z)}=\tau_{k} G_{+}(z)$. But $\left|\tau_{k} G_{+}(z)\right|>1$ according to (49). Therefore,

$$
\left(\phi_{0}(z), \phi_{1}(z), \ldots, \phi_{n}(z), \ldots\right) \notin \ell^{2}
$$

which contradicts (63). Hence, for all $z \in \operatorname{supp}(\mu) \backslash\left[\bigcup_{s=1}^{k}\left\{z: \Delta_{k-1}^{(s)}(z)=0\right\} \cup(\operatorname{supp}(\mu))^{\prime}\right]$ and all $j=1, \ldots, k$

$$
\lim _{n \longrightarrow \infty} \frac{\Phi_{n+k}(z)}{\Phi_{n}(z)}=G_{-}(z) .
$$

This proves that $i$ ) in Theorem 3 implies $i$ ii). Since $i i$ ) is a trivial consequence of $i i i$ ), with this we conclude the proof of Theorem 3. Let us proceed with the proof of Theorem 2 .

Now, we know that for $z \in \Omega \backslash \operatorname{supp}(\mu)$

$$
\lim _{n \longrightarrow \infty} \frac{\Phi_{n+k}(z)}{\Phi_{n}(z)}=G_{+}(z) .
$$

In order to prove that this limit is uniform on each compact subset of $\Omega \backslash \operatorname{supp}(\mu)$ it suffices to show that the family $\left\{\frac{\Phi_{n+k}(z)}{\Phi_{n}(z)}\right\}, n=\nu k+j, j$ fixed, is normal for all sufficiently large $\nu$; that is, uniformly bounded on each compact subset of the given region $\Omega \backslash \operatorname{supp}(\mu)$. We do this by showing that if $z_{0} \in \Omega \backslash \operatorname{supp}(\mu)$, then on a sufficiently small neighborhood 
$D\left(\varepsilon ; z_{0}\right)=\left\{z:\left|z-z_{0}\right|<\varepsilon\right\}$ the given family is uniformly bounded for all sufficiently large $\nu$. In the sequel $j=1, \ldots, k$ and $z_{0} \in \Omega \backslash \operatorname{supp}(\mu)$ are fixed, $n=\nu k+j$. There exists a closed neighborhood $B$ of $z_{0}$ contained in $\Omega \backslash \operatorname{supp}(\mu)$ and $\nu_{0}$ such that for all $z \in B$ and $\nu \geq \nu_{0}$ the three term recurrence relation (53) takes place. We only consider such $z$ and $\nu$.

Whenever $\phi_{n-k}(z) \neq 0 \neq \phi_{n}(z)$ formula (53) may be rewritten as follows

$$
\frac{\phi_{n+k}(z)}{\phi_{n}(z)}=\frac{\kappa_{n+k}}{\kappa_{n}} \alpha_{n, 1}(z)-\frac{\kappa_{n+k}}{\kappa_{n-k}} \alpha_{n, 2}(z) \frac{1}{\frac{\phi_{n}(z)}{\phi_{n-k}(z)}} .
$$

At point $z_{0}$ this relation also takes place for all sufficiently large $\nu$. This is a consequence of the fact that

$$
\lim _{\nu} \frac{\phi_{n+k}\left(z_{0}\right)}{\phi_{n}\left(z_{0}\right)}=\tau_{k} G_{+}\left(z_{0}\right) \neq 0
$$

because then either $\phi_{n}\left(z_{0}\right) \neq 0$ for all sufficiently large $\nu$ or $z_{0}$ is a zero of equal multiplicity of all the polynomials for all sufficiently large $\nu$ which serves the same purpose. Notice that

$$
\tau_{k} G_{+}(z)+\tau_{k} G_{-}(z)=\tau_{k} p(z), \tau_{k} G_{+}(z) \tau_{k} G_{-}(z)=z^{k} \frac{\Psi_{k+1}(0)}{\Psi_{1}(0)} .
$$

Therefore,

$$
\begin{aligned}
\frac{\phi_{n+k}(z)}{\phi_{n}(z)}-\tau_{k} G_{+}\left(z_{0}\right)= & \frac{\kappa_{n+k}}{\kappa_{n}} \alpha_{n, 1}(z)-\tau_{k} p\left(z_{0}\right)+\frac{\kappa_{n+k}}{\kappa_{n-k}} \alpha_{n, 2}(z)\left(\frac{1}{\tau_{k} G_{+}\left(z_{0}\right)}-\frac{1}{\frac{\phi_{n}(z)}{\phi_{n-k}(z)}}\right) \\
& +\frac{1}{\tau_{k} G_{+}\left(z_{0}\right)}\left(\frac{\Psi_{k+1}(0)}{\Psi_{1}(0)} z_{0}^{k}-\frac{\kappa_{n+k}}{\kappa_{n-k}} \alpha_{n, 2}(z)\right) .
\end{aligned}
$$

Take $\rho>0$ such that $\rho<\min \left\{1-\left|\tau_{k} G_{-}\left(z_{0}\right)\right|,\left|\tau_{k} G_{+}\left(z_{0}\right)\right|-1\right\}$ (see (49)). We have

$$
\lim _{\nu} \frac{\kappa_{n+k}}{\kappa_{n}} \alpha_{n, 1}(z)=\tau_{k} p(z), \lim _{\nu} \frac{\kappa_{n+k}}{\kappa_{n-k}} \alpha_{n, 2}(z)=z^{k} \frac{\Psi_{k+1}(0)}{\Psi_{1}(0)}
$$

uniformly on $B$. Because of this and the continuity of the limit functions, there exists $\varepsilon_{1}>0$ and $\nu_{1} \in \mathbb{N}$ such that for all $z \in D\left(\varepsilon_{1} ; z_{0}\right) \subset B$ and $\nu \geq \nu_{1}$

$$
\begin{gathered}
\left|\frac{\kappa_{n+k}}{\kappa_{n}} \alpha_{n, 1}(z)-\tau_{k} p\left(z_{0}\right)\right|<\frac{1-\left(\left|\tau_{k} G_{-}\left(z_{0}\right)\right|+\rho\right)}{2} \rho, \\
\left|\frac{\kappa_{n+k}}{\kappa_{n-k}} \alpha_{n, 2}(z)-z_{0}^{k} \frac{\Psi_{k+1}(0)}{\Psi_{1}(0)}\right|<\frac{1-\left(\left|\tau_{k} G_{-}\left(z_{0}\right)\right|+\rho\right)}{2} \rho,
\end{gathered}
$$

and

$$
\left|\frac{\frac{\kappa_{n+k}}{\kappa_{n-k}} \alpha_{n, 2}(z)}{\tau_{k} G_{+}\left(z_{0}\right)}\right|<\left|\tau_{k} G_{-}\left(z_{0}\right)\right|+\rho .
$$


Using (65)-(68), we obtain that for all $z \in D\left(\varepsilon_{1} ; z_{0}\right)$ and $\nu \geq \nu_{1}$ such that $\phi_{n-k}(z) \neq 0 \neq$ $\phi_{n}(z)$

$$
\begin{aligned}
\left|\frac{\phi_{n+k}(z)}{\phi_{n}(z)}-\tau_{k} G_{+}\left(z_{0}\right)\right| & <\left[1-\left(\left|\tau_{k} G_{-}\left(z_{0}\right)\right|+\rho\right)\right] \rho \\
& +\left(\left|\tau_{k} G_{-}\left(z_{0}\right)\right|+\rho\right)\left|\frac{\frac{\phi_{n}(z)}{\phi_{n-k}(z)}-\tau_{k} G_{+}\left(z_{0}\right)}{\frac{\phi_{n}(z)}{\phi_{n-k}(z)}}\right| .
\end{aligned}
$$

Following the arguments above, we chose $\nu_{1}$ so that (69) takes place at $z=z_{0}$ for all $\nu \geq \nu_{1}$ even if this point is a zero of infinitely many polynomials $\phi_{n}$.

Since $\lim _{\nu} \frac{\phi_{n}\left(z_{0}\right)}{\phi_{n-k}\left(z_{0}\right)}=\tau_{k} G_{+}\left(z_{0}\right)$. Choose and fix $\nu_{2} \geq \nu_{1}$ such that

$$
\left|\frac{\phi_{n_{2}}\left(z_{0}\right)}{\phi_{n_{2}-k}\left(z_{0}\right)}-\tau_{k} G_{+}\left(z_{0}\right)\right|<\rho .
$$

Using the analyticity of $\phi_{n_{2}-k}(z), \phi_{n_{2}}(z)$, and the continuity of $\frac{\phi_{n_{2}}(z)}{\phi_{n_{2}-k}(z)}$, we can find $0<\varepsilon_{2}<\varepsilon_{1}$ such that for all $z \in D\left(\varepsilon_{2} ; z_{0}\right) \backslash\left\{z_{0}\right\}, \phi_{n_{2}-k}(z) \neq 0 \neq \phi_{n_{2}}(z)$ and

$$
\left|\frac{\phi_{n_{2}}(z)}{\phi_{n_{2}-k}(z)}-\tau_{k} G_{+}\left(z_{0}\right)\right|<\rho .
$$

For $z \in D\left(\varepsilon_{2} ; z_{0}\right)$, from (70) and the definition of $\rho$, we get

$$
\left|\frac{\phi_{n_{2}}(z)}{\phi_{n_{2}-k}(z)}\right|>\left|\tau_{k} G_{+}\left(z_{0}\right)\right|-\rho>1 .
$$

Combining (69)-(71), we obtain

$$
\left|\frac{\phi_{n_{2}+k}(z)}{\phi_{n_{2}}(z)}-\tau_{k} G_{+}\left(z_{0}\right)\right|<\rho, z \in D\left(\varepsilon_{2} ; z_{0}\right),
$$

which is (70) for $\nu=\nu_{2}+1$. This inequality implies that

$$
\left|\frac{\phi_{n_{2}+k}(z)}{\phi_{n_{2}}(z)}\right|>\left|\tau_{k} G_{+}\left(z_{0}\right)\right|-\rho>1, z \in D\left(\varepsilon_{2} ; z_{0}\right)
$$

which is (71) for $\nu=\nu_{2}+1$. In particular, we obtain that $\phi_{n_{2}+k}(z) \neq 0$ for all $z \in$ $D\left(\varepsilon_{2} ; z_{0}\right) \backslash\left\{z_{0}\right\}$. We are now in condition of using (69) for the index $\nu_{2}+1$ obtaining

$$
\left|\frac{\phi_{n_{2}+2 k}(z)}{\phi_{n_{2}+k}(z)}-\tau_{k} G_{+}\left(z_{0}\right)\right|<\rho, z \in D\left(\varepsilon_{2} ; z_{0}\right) .
$$

Repeating this process consecutively for all $\nu \geq \nu_{2}$, we obtain that

$$
\left|\frac{\phi_{n+k}(z)}{\phi_{n}(z)}-\tau_{k} G_{+}\left(z_{0}\right)\right|<\rho, z \in D\left(\varepsilon_{2} ; z_{0}\right)
$$

for all $\nu \geq \nu_{2}, n=\nu k+j$. In other words,

$$
\frac{\phi_{n+k}(z)}{\phi_{n}(z)} \in D\left(\rho ; \tau_{k} G_{+}\left(z_{0}\right)\right), z \in D\left(\varepsilon_{2} ; z_{0}\right), \nu \geq \nu_{2},
$$


which is what we needed to prove.

Therefore, we have obtained uniform convergence in (51) on each compact subset of $\Omega \backslash \operatorname{supp}(\mu)$ as stated in Theorem 2. The other limit relations in (51) are a trivial consequence of the definition of $M_{\Gamma}\left(a_{1}, \ldots, a_{k} ; b_{1}, \ldots, b_{k}\right)$ and (8).

That $(\operatorname{supp}(\mu))^{\prime}=\gamma(\bar{\mu})$ was proved in Lemma 9, and the fact that on any compact subset $K$ of $\Omega \backslash \operatorname{supp}(\mu)$ there are no zeros of the polynomials $\phi_{n}(z)$ for all sufficiently large $n$ is a consequence of (72) and the compactness principle. The proof of Theorem 2 is concluded.

2. Before stating the next result let us introduce some new notation. As above $\mu$ is a probability measure on $\Gamma$ and $\left\{\Phi_{n}(z)\right\}$, the corresponding sequence of monic orthogonal polynomials. Set

$$
F_{\mu}(z)=F(z)=\int \frac{\zeta+z}{\zeta-z} d \mu(\zeta)
$$

the associated Caratheodory function, and

$$
\Theta_{n}(z)=\int \frac{\zeta+z}{\zeta-z}\left[\Phi_{n}(\zeta)-\Phi_{n}(z)\right] d \mu(\zeta)
$$

the $n$-th degree second kind monic polynomial. From (73), we have that

$$
\Theta_{n}(0)=-\Phi_{n}(0) .
$$

Therefore, if $\mu \in M_{\Gamma}\left(a_{1}, \ldots, a_{k} ; b_{1}, \ldots, b_{k}\right)$, it follows that $\left\{\Theta_{n}(z)\right\}$ is a sequence of monic orthogonal polynomials with respect to a measure $\mu_{1} \in M_{\Gamma}\left(a_{1}, \ldots, a_{k} ; b_{1}, \ldots, b_{k}\right)$. Denote $\theta_{n}(z)$ the $n$-th orthonormal polynomial with respect to the probability measure $\mu_{1}$. From (8) and (74), we have that

$$
\theta_{n}(z)=\kappa_{n} \Theta_{n}(z)
$$

where $\kappa_{n}$ is the leading coefficient of the $n$-th orthonormal polynomials $\phi_{n}$ with respect to $\mu$.

The following formulas are well-known (see e.g. (1.17) and (1.19) in [10])

$$
\theta_{n}(z) \phi_{n}^{*}(z)+\phi_{n}(z) \theta_{n}^{*}(z)=2 z^{n}
$$

and

$$
F(z) \phi_{n}(z)+\theta_{n}(z)=\int \frac{\zeta+z}{\zeta-z} \phi_{n}(\zeta) d \mu(\zeta)
$$

where, as usual, $\phi_{n}^{*}, \theta_{n}^{*}$ denote the reversed polynomials. In particular, (77) is a consequence of (73) and (75).

Lemma 10. Let $\mu$ be a probability measure for which Szegö's condition is not satisfied. Then

$$
\sigma_{p}(U(\mu)) \cap \sigma_{p}\left(U\left(\mu_{1}\right)\right)=\emptyset .
$$

Moreover, if $\mu \in M_{\Gamma}\left(a_{1}, \ldots, a_{k} ; b_{1}, \ldots, b_{k}\right)$ then

$$
\gamma(\bar{\mu})=\left(\operatorname{supp}\left(\mu_{1}\right)\right)^{\prime}
$$

and

$$
\left[\operatorname{supp}(\mu) \cap \operatorname{supp}\left(\mu_{1}\right)\right] \backslash \gamma(\bar{\mu})=\emptyset .
$$


Proof. It is well known that Szegö's condition is satisfied if and only if

$$
\sum_{n=0}^{\infty}\left|\Phi_{n}(0)\right|^{2}<+\infty
$$

Using (74) we get that $\mu_{1}$ doesn't satisfy Szegö's condition. Therefore, the spectra of $U\left(\mu_{1}\right)$ and $U(\mu)$ are contained in $\Gamma$. Let $z \in \sigma_{p}(U(\mu)) \cap \sigma_{p}\left(U\left(\mu_{1}\right)\right)$, then $|z|=1$. From Lemma 1 and (76), using the Cauchy-Schwartz inequality we arrive to a contradiction. Notice that $\left|\phi_{n}^{*}(z)\right|=\left|\phi_{n}(z)\right|$ and $\left|\theta_{n}^{*}(z)\right|=\left|\theta_{n}(z)\right|$ when $|z|=1$. Thus (78) takes place.

We already pointed out that in case that $\mu \in M_{\Gamma}\left(a_{1}, \ldots, a_{k} ; b_{1}, \ldots, b_{k}\right)$ then $\mu_{1} \in$ $M_{\Gamma}\left(a_{1}, \ldots, a_{k} ; b_{1}, \ldots, b_{k}\right)$; therefore, (79) follows from Theorem 2. Finally, (79) and Lemma 1 tell us that $\left[\operatorname{supp}(\mu) \cap \operatorname{supp}\left(\mu_{1}\right)\right] \backslash \gamma(\bar{\mu})$ may only contain isolated mass points belonging to the spectra of $U(\mu)$ and $U\left(\mu_{1}\right)$, but such points do not exist according to (78). Hence (80) takes place.

Theorem 4. Let $\mu \in M_{\Gamma}\left(a_{1}, \ldots, a_{k} ; b_{1}, \ldots, b_{k}\right)$. Then

$$
\varlimsup_{n \longrightarrow \infty}\left\|\frac{\theta_{n}(z)}{\phi_{n}(z)}+F(z)\right\|_{K}^{1 / n} \leq\left(\inf _{K}\left|\tau_{k} G_{+}(z)\right|\right)^{-1 / k}<1
$$

and

$$
\varlimsup_{n \longrightarrow \infty}\left\|\frac{\theta_{n}^{*}(z)}{\phi_{n}^{*}(z)}-F(z)\right\|_{K}^{1 / n} \leq\left(\inf _{K}\left|\tau_{k} G_{+}(z)\right|\right)^{-1 / k}<1
$$

where $K$ is any compact subset of $\overline{\mathbb{C}} \backslash\left(\bigcup_{s=1}^{k}\left\{z: \Delta_{k-1}^{(s)}(z)=0\right\} \cup \operatorname{supp}(\mu)\right)$. Moreover, let $z_{0}$ be an isolated point of $\operatorname{supp}(\mu)$. Then, for all sufficiently small $r>0$ there exists $n_{0}$ such that for all $n \geq n_{0}, \phi_{n}$ has exactly one zero contained in $\left\{z:\left|z-z_{0}\right|<r\right\}$.

Proof. As an immediate consequence of (51), we obtain that

$$
\lim _{n \longrightarrow \infty}\left|\phi_{n}(z)\right|^{1 / n}=\left|\tau_{k} G_{+}(z)\right|^{1 / k}
$$

uniformly on each compact subset $K \subset \mathbb{C} \backslash\left(\bigcup_{s=1}^{k}\left\{z: \Delta_{k-1}^{(s)}(z)=0\right\} \cup \operatorname{supp}(\mu)\right)$. On the other hand, from (77), we have

$$
\begin{aligned}
\left|\frac{\theta_{n}(z)}{\phi_{n}(z)}+F(z)\right| & =\left|\frac{1}{\phi_{n}(z)} \int \frac{\zeta+z}{\zeta-z} \phi_{n}(\zeta) d \mu(\zeta)\right| \leq \\
& \leq \frac{1}{\left|\phi_{n}(z)\right|}\left(\int\left|\frac{\zeta+z}{\zeta-z}\right|^{2} d \mu(\zeta)\right)^{1 / 2}\left(\int\left|\phi_{n}(\zeta)\right|^{2} d \mu(\zeta)\right)^{1 / 2} \leq \\
& \leq \frac{c_{1}}{\left|\phi_{n}(z)\right|}
\end{aligned}
$$

where

$$
c_{1}=c_{1}(K)=\sup _{z \in K}\left(\int\left|\frac{\zeta+z}{\zeta-z}\right|^{2} d \mu(\zeta)\right)^{1 / 2}<+\infty .
$$

Relation (81) follows immediately from (83)-(84). Since $\frac{\theta_{n}(z)}{\phi_{n}(z)}+F(z)$ is holomorphic at $\infty$, using the maximum principle, we have that (81) is also true on any compact subset $K \subset$ 
$\overline{\mathbb{C}} \backslash\left(\bigcup_{s=1}^{k}\left\{z: \Delta_{k-1}^{(s)}(z)=0\right\} \cup \operatorname{supp}(\mu)\right) .(81)$ and (82) are equivalent from the definition of reversed polynomial and the fact that $\overline{F(1 / \bar{z})}=-F(z)$.

Take $z_{0}$ an isolated point in $\operatorname{supp}(\mu)$. That is $z_{0} \in \operatorname{supp}(\mu) \backslash \gamma(\bar{\mu})$. From $(80)$, we have that $z_{0} \notin \operatorname{supp}\left(\mu_{1}\right)$. Therefore, the distance $d$ from $z_{0}$ to the convex hull $\operatorname{conv}\left(\operatorname{supp}\left(\mu_{1}\right)\right)$ of $\operatorname{supp}\left(\mu_{1}\right)$ is greater than zero. Take $r>0$ sufficiently small so that $\left\{z:\left|z-z_{0}\right| \leq r\right\}$ contains no point from the set

$$
\left[\bigcup_{s=1}^{k}\left\{z: \Delta_{k-1}^{(s)}(z)=0\right\} \cup \operatorname{conv}\left(\operatorname{supp}\left(\mu_{1}\right)\right) \cup \operatorname{supp}(\mu)\right] \backslash\left\{z_{0}\right\} .
$$

It is well known that the zeros of orthogonal polynomials lie in the convex hull of the support of the orthogonality measure. Thus, for all $n \in \mathbb{Z}_{+}, \theta_{n}$ has no zero in $\left\{z:\left|z-z_{0}\right| \leq r\right\}$. Take $K=\left\{z:\left|z-z_{0}\right|=r\right\}$, from (81), we know that

$$
\lim _{n \longrightarrow \infty} \frac{\theta_{n}(z)}{\phi_{n}(z)}=-F(z)
$$

uniformly on $K$ and $F(z) \neq 0, z \in K$ (the zeros of $F(z)=F_{\mu}(z)$ are the poles of $F_{\mu_{1}}(z)$, since $\left.F_{\mu_{1}}(z)=\frac{1}{F_{\mu}(z)}\right)$. Therefore, by the argument principle,

$$
\lim _{n \longrightarrow \infty} \frac{1}{2 \pi} \int_{K} \frac{\left(\frac{\theta_{n}(z)}{\phi_{n}(z)}\right)^{\prime}}{\frac{\theta_{n}(z)}{\phi_{n}(z)}} d z=\frac{1}{2 \pi} \int_{K} \frac{F^{\prime}(z)}{F(z)} d z=-1
$$

because $F$ has no zero in $\left\{z:\left|z-z_{0}\right|<r\right\}$ and exactly a simple pole at $z_{0}$ since it is an isolated mass point of $\mu$. The left-hand of (85) equals, for all sufficiently large $n$, minus the number of zeros that $\phi_{n}$ has inside of $K$ (since $\theta_{n}$ has none). These integers according to $(85)$ tend to -1 ; therefore, for all sufficiently large $n$ must equal -1 . Hence we have proved the final statement of Theorem 4.

Remark 1. For measures in $M_{\Gamma}\left(a_{1}, \ldots, a_{k} ; b_{1}, \ldots, b_{k}\right)$, the asymptotic behavior of the zeros is well described by Theorems 2 and 4. Each point in $\operatorname{supp}(\mu) \backslash \gamma(\bar{\mu})$ "attracts" exactly one zero. The rest go to

$$
\bigcup_{s=1}^{k}\left\{z: \Delta_{k-1}^{(s)}(z)=0\right\} \cup \gamma(\bar{\mu})
$$

The points in $\bigcup_{s=1}^{k}\left\{z: \Delta_{k-1}^{(s)}(z)=0\right\}$ may attract zeros or not. It is easy to see using the argument principle for the sequence $\left\{\frac{\Phi_{n+k}}{\Phi_{n}}\right\}, n=k \nu+j, \nu \in \mathbb{N}$, that for each fixed $j=1, \ldots, k$, there exists $r>0$ sufficiently small such that for all sufficiently large $\nu$ the amount of zeros of these polynomials inside the neighborhood of radius $r$ of a point in $\bigcup_{s=1}^{k}\left\{z: \Delta_{k-1}^{(s)}(z)=0\right\}$ is fixed. 


\section{Corollaries}

1. From Theorems 2 and 3 , we obtain

Corollary 1. Let $\mu \in M_{\Gamma}\left(a_{1}, \ldots, a_{k} ; b_{1}, \ldots, b_{k}\right)$. Then

$$
\lim _{n=j \bmod k} \frac{\Phi_{n+1}(z)}{\Phi_{n}(z)}=\left[\Delta_{k}^{(j+1)}(z)-G_{-}(z)\right] \frac{1}{\Delta_{k-1}^{(j+1)}(z)}
$$

uniformly on each compact subset of $\mathbb{C} \backslash\left(\bigcup_{s=1}^{k}\left\{z: \Delta_{k-1}^{(s)}(z)=0\right\} \cup \operatorname{supp}(\mu)\right)$ and

$$
\lim _{n=j \bmod k} \frac{\Phi_{n+1}(z)}{\Phi_{n}(z)}=\left[\Delta_{k}^{(j+1)}(z)-G_{+}(z)\right] \frac{1}{\Delta_{k-1}^{(j+1)}(z)}
$$

for each $z \in \operatorname{supp}(\mu) \backslash\left(\bigcup_{s=1}^{k}\left\{z: \Delta_{k-1}^{(s)}(z)=0\right\} \cup \gamma(\bar{\mu})\right)$.

Proof. We must simply use (58) and (51) or (52) respectively in case that we wish to prove $(86)$ or $(87)$.

Remark 2. Since $\frac{\kappa_{n}}{\kappa_{n+1}}=\sqrt{1-\left|\Phi_{n+1}(0)\right|^{2}}$ and $\lim _{n=j \bmod k}\left|\Phi_{n+1}(0)\right|=a_{j+1}$ the corresponding asymptotic formulas for the orthonormal polynomials follow immediately. On the other hand,

$$
\Delta_{k}^{(j+1)}(z)-z \frac{\Psi_{j+2}(0)}{\Psi_{j+1}(0)}\left(\frac{\tau_{j}}{\tau_{j+1}}\right)^{2} \Delta_{k-2}^{(j+2)}(z)=p(z) \quad \text { and } \quad G_{+}(z)+G_{-}(z)=p(z) ;
$$

therefore,

$$
\Delta_{k}^{(j+1)}(z)-G_{-}(z)=z \frac{\Psi_{j+2}(0)}{\Psi_{j+1}(0)}\left(\frac{\tau_{j}}{\tau_{j+1}}\right)^{2} \Delta_{k-2}^{(j+2)}(z)+G_{+}(z)
$$

and

$$
\Delta_{k}^{(j+1)}(z)-G_{+}(z)=z \frac{\Psi_{j+2}(0)}{\Psi_{j+1}(0)}\left(\frac{\tau_{j}}{\tau_{j+1}}\right)^{2} \Delta_{k-2}^{(j+2)}(z)+G_{-}(z) .
$$

These identities may be used for giving a different (equivalent) expression for the righthand sides of (86) and (87).

In the next result $f^{(m)}$ denotes the $m$-th derivate of $f$.

Corollary 2. Let $\mu \in M_{\Gamma}\left(a_{1}, \ldots, a_{k} ; b_{1}, \ldots, b_{k}\right)$. Then for each fixed $m \in \mathbb{Z}_{+}$

$$
\begin{gathered}
\lim _{n \longrightarrow \infty} \frac{\Phi_{n+k}^{(m)}(z)}{\Phi_{n}^{(m)}(z)}=G_{+}(z), \\
\lim _{n \longrightarrow \infty}\left|\Phi_{n}^{(m)}(z)\right|^{k / n}=\left|G_{+}(z)\right|,
\end{gathered}
$$




$$
\lim _{n \longrightarrow \infty} \frac{k \Phi_{n}^{(m+1)}(z)}{n \Phi_{n}^{(m)}(z)}=\frac{G_{+}^{(1)}(z)}{G_{+}(z)}
$$

and

$$
\lim _{n \longrightarrow \infty} \frac{n}{k}\left(\frac{\Phi_{n+k}^{(m+1)}(z)}{\Phi_{n}^{(m+1)}(z)}-\frac{\Phi_{n+k}^{(m)}(z)}{\Phi_{n}^{(m)}(z)}\right)=G_{+}(z),
$$

uniformly on each compact subset of $\mathbb{C} \backslash\left(\bigcup_{s=1}^{k}\left\{z: \Delta_{k-1}^{(s)}(z)=0\right\} \cup \operatorname{supp}(\mu)\right)$.

Proof. The proof of this Corollary follows the same line of reasoning as for the proof of Corollary 6 in [1] so we will only sketch the proof.

One starts with $m=0$ for which (88) is the main statement of Theorem 2. Then, (89) follows easily from (88). In order to prove (90), notice that $\left\{\frac{k}{n} \frac{\Phi_{n}^{(1)}(z)}{\Phi_{n}(z)}\right\}, n \geq 0$, is normal in $\mathbb{C} \backslash\left[\bigcup_{s=1}^{k}\left\{z: \Delta_{k-1}^{(s)}(z)=0\right\} \cup \operatorname{supp}(\mu)\right]$. This is an immediate consequence of the formula

$$
\frac{k}{n} \frac{\Phi_{n}^{(1)}(z)}{\Phi_{n}(z)}=\frac{k}{n} \sum_{j=1}^{n} \frac{1}{z-z_{n, j}},
$$

where $z_{n, 1}, \ldots, z_{n, n}$ are the $n$ (not necessarily distinct) zeros of $\Phi_{n}$, and the fact that given a compact set $K \subset \mathbb{C} \backslash\left[\bigcup_{j=1}^{k}\left\{z: \Delta_{k-1}^{(s)}(z)=0\right\} \cup \operatorname{supp}(\mu)\right]$, for all sufficiently large $n$, the zeros of $\Phi_{n}$ are bounded away from $K$. Thus in order to prove (90) it suffices to show that on a segment $[c, d] \subset \mathbb{R} \backslash\left[\bigcup_{j=1}^{k}\left\{z: \Delta_{k-1}^{(s)}(z)=0\right\} \cup \operatorname{supp}(\mu)\right]$

$$
\lim _{n \longrightarrow \infty} \operatorname{Re}\left(\frac{k}{n} \frac{\Phi_{n}^{(1)}(x)}{\Phi_{n}(x)}\right)=\operatorname{Re}\left(\frac{G_{+}^{(1)}(x)}{G_{+}(x)}\right), x \in[c, d] .
$$

Now, take into consideration that

$$
\operatorname{Re}\left(\frac{k}{n} \frac{\Phi_{n}^{(1)}(x)}{\Phi_{n}(x)}\right)=\frac{k}{2 n} \sum_{j=1}^{k}\left(\frac{1}{x-z_{n, k}}+\frac{1}{x-\bar{z}_{n, k}}\right)=\left(\frac{k}{2 n} \log \left|\phi_{n}(x)\right|^{2}\right)_{x}^{(1)}
$$

where $(\cdot)_{x}^{(1)}$ denotes the derivate with respect to $x$.

On the other hand, from (89), we have

$$
\lim _{n \longrightarrow \infty}\left(\frac{k}{n} \log \left|\phi_{n}(x)\right|\right)_{x}^{(1)}=\left(\log \left|G_{+}(x)\right|\right)_{x}^{(1)}=\operatorname{Re}\left(\log G_{+}(x)\right)_{x}^{(1)}=\operatorname{Re}\left(\frac{G_{+}^{(1)}(x)}{G_{+}(x)}\right) .
$$

From (94) and (93), we obtain (92) as needed.

Using (88), (90) (for $m=0$ ) and the identity

$$
n \frac{\Phi_{n}(z)}{\Phi_{n}^{(1)}(z)}\left(\frac{\Phi_{n+k}(z)}{\Phi_{n}(z)}\right)^{(1)}=n\left(\frac{\Phi_{n+k}^{(1)}(z)}{\Phi_{n}^{(1)}(z)}-\frac{\Phi_{n+k}(z)}{\Phi_{n}(z)}\right),
$$


we get (91) for $m=0$ which in turn implies (88) for $m=1$. We are able to repeat the process for $m=1$ and so forth for each $m \in \mathbb{Z}_{+}$.

Remark 3. Theorems 2-4 and Corollaries 1-2 yield analogous results for the corresponding relations when the reversed polynomials are considered.

2. Let us prove Theorem 1. Obviously, (6) implies that $\mu \in M_{\Gamma}(a ; b)$. The reversed implication and the other statements of Theorem 1 , when $a \in(0,1)$, are contained in Theorems 2-4. Notice that for $k=1,\left\{z: \Delta_{k-1}^{(1)}(z)=0\right\}=\emptyset$ since $\Delta_{0}^{(1)}(z) \equiv 1$.

When $a=1$, the existence of

$$
\lim _{n \longrightarrow \infty} \frac{\kappa_{n}}{\kappa_{n+1}}=\lim _{n \longrightarrow \infty} \sqrt{1-\left|\Phi_{n+1}(0)\right|^{2}}=0
$$

is immediate. Nonetheless, our proof above of ratio asymptotics encounters some difficulties. The main one is that we can no longer construct a representative measure of type $\bar{\mu}$ for the class $M_{\Gamma}(a ; b)$ as we did in Lemma 6 (or $\widehat{\mu}$ of Lemma 7). This is so because from $(5),|b|=1$, and $\left\{b^{n}\right\}$ is no longer a sequence of reflection coefficients. On the other hand, in this particular case things simplify quite a bit.

First of all notice that for $\mu \in M_{\Gamma}(1 ; b)$

$$
U(\mu)+b I
$$

(where $I$ is the identity) is compact; therefore, by Weyl's Theorem

$$
\operatorname{supp}(\mu)=\sigma(U)=\{-b\} \cup(\operatorname{supp}(\mu) \backslash\{-b\})
$$

where $\operatorname{supp}(\mu) \backslash\{-b\}$ consists of at most a denumerable set of isolated points in $\Gamma \backslash\{-b\}$.

Secondly, from (16), we have

$$
0=\Phi_{n+1}(z)-\left(z+\frac{\Phi_{n+1}(0)}{\Phi_{n}(0)}\right) \Phi_{n}(z)+z \frac{\kappa_{n-1}^{2}}{\kappa_{n}^{2}} \frac{\Phi_{n+1}(0)}{\Phi_{n}(0)} \Phi_{n-1}(z), n \geq 1 .
$$

In applying Poincaré's Theorem, from (2) and (95) the corresponding characteristic equation is

$$
0=\lambda^{2}-(z+b) \lambda
$$

whose roots are

$$
G_{+}(z)=\lambda_{+}=z+b ; G_{-}(z)=\lambda_{-}=0 .
$$

Therefore

$$
\left|G_{+}(z)\right| \neq\left|G_{-}(z)\right| \Longleftrightarrow G_{+}(z) \neq G_{-}(z)=0 \Longleftrightarrow z \neq-b .
$$

The proof continues along the same lines from this point on. We leave the details to the reader.

Corollary 3. Let $\mu \in M_{\Gamma}(a ; b), a \in(0,1]$, then

$$
\lim _{n \longrightarrow \infty} \frac{\Phi_{n+1}^{*}(z)}{\Phi_{n}^{*}(z)}=\overline{z G_{+}\left(\frac{1}{\bar{z}}\right)}=\bar{b} G_{+}(z)
$$


and

$$
\lim _{n \longrightarrow \infty} \Phi_{n+1}(0) \frac{\Phi_{n}^{*}(z)}{\Phi_{n}(z)}=G_{+}(z)-z,
$$

uniformly on each compact subset of $\mathbb{C} \backslash \operatorname{supp}(\mu)$. Also

$$
\lim _{n \longrightarrow \infty} \frac{\Phi_{n+1}^{*}(z)}{\Phi_{n}^{*}(z)}=\overline{z G_{-}\left(\frac{1}{\bar{z}}\right)}=\bar{b} G_{-}(z), z \in \operatorname{supp}(\mu) \backslash \gamma(\bar{\mu}),
$$

where $\gamma(\bar{\mu})=\{-b\}$ in case that $a=1$.

The proof may be carried out using the same scheme as for Corollary 1 in [4].

Remark 4. Theorems 2-4 may be extended to the case when $\mu \in M_{\Gamma}\left(a_{1}, \ldots, a_{k} ; b_{1}, \ldots, b_{k}\right)$ and for some $i \in\{1, \ldots, k\}, a_{i}=1$. A representative $\bar{\mu}$ does not exist, but it is not hard to show that in this case

$$
\operatorname{supp}(\mu)=\{z: p(z)=0\} \cup(\operatorname{supp}(\mu) \backslash\{z: p(z)=0\})
$$

and $\operatorname{supp}(\mu) \backslash\{z: p(z)=0\}$ consists of at most a denumerable set of isolated points in $\Gamma \backslash\{z: p(z)=0\}$. Moreover,

$$
p(z)=\Delta_{k}^{(i)}(z)
$$

where $\Delta_{k}^{(i)}(z)$ is the determinant constructed with the limit values given by (5). There is no problem in using Poincaré's Theorem to get the corresponding result on ratio asymptotics. Here, the characteristic equation is

$$
0=\lambda^{2}-p(z) \lambda,
$$

and

$$
G_{+}(z)=p(z), G_{-}(z)=0 .
$$

For the class $M_{\Gamma}(1, b)$, we have that $p(z)=z+b$. In [12, Th. 6], it was proved that the structure of $\operatorname{supp}(\mu)$ shown above when $p(z)=z+b$ is not only a necessary but also a sufficient condition for $\mu$ to be in $M_{\Gamma}(1, b)$. It would interesting to prove that this is true in general for $\mu \in M_{\Gamma}\left(a_{1}, \ldots, a_{k} ; b_{1}, \ldots, b_{k}\right)$ and for some $i \in\{1, \ldots, k\}, a_{i}=1$.

Let

$$
K_{n}(z, \zeta)=\sum_{k=0}^{n-1} \phi_{k}(z) \overline{\phi_{k}(\zeta)}
$$

and

$$
\omega_{n}(z)=K_{n}^{-1}(z, z),
$$

where $\phi_{n}$ denotes the $n$-th orthonormal polynomial.

Corollary 4. Let $\mu \in M_{\Gamma}\left(a_{1}, \ldots, a_{k} ; b_{1}, \ldots, b_{k}\right), a_{j} \in(0,1], j=1,2, \ldots, k$. Then

$$
\lim _{n=j \bmod k} \frac{K_{n}(z, \zeta)}{\phi_{n}(z) \overline{\phi_{n}(\zeta)}}=\frac{1}{1-z \bar{\zeta}}\left[\frac{\left(\eta_{j}(z)-z\right) \overline{\left(\eta_{j}(\zeta)-\zeta\right)}}{a_{j+1}^{2}}-1\right], j=1,2, \ldots, k,
$$


uniformly on each compact subset of $\left[\overline{\mathbb{C}} \backslash\left(\bigcup_{s=1}^{k}\left\{\xi: \Delta_{k-1}^{(s)}(\xi)=0\right\} \cup \operatorname{supp}(\mu)\right)\right]^{2}$, where

$$
\eta_{j}(\xi)=\frac{\Delta_{k}^{(j+1)}(\xi)-G_{-}(\xi)}{\Delta_{k-1}^{(j+1)}(\xi)}, j=1,2, \ldots, k .
$$

Moreover, if $a_{j}=1$ then the right hand of (96) is 0.

In particular, if $\mu \in M_{\Gamma}(a ; b), a \in(0,1]$, we have

$$
\lim _{n \longrightarrow \infty} \frac{K_{n}(z, \zeta)}{\phi_{n}(z) \overline{\phi_{n}(\zeta)}}=\frac{C^{2}(\gamma)}{G_{+}(z) \overline{G_{+}(\zeta)}-C^{2}(\gamma)},
$$

uniformly on each compact subset of $(\overline{\mathbb{C}} \backslash \operatorname{supp}(\mu))^{2}$, where $\gamma=\gamma(\bar{\mu})$ and $C(\gamma)$ denotes the logarithmic capacity of $\gamma$. In this case we have

$$
\lim _{n \longrightarrow \infty} \frac{1}{\omega_{n}(z)\left|\phi_{n}(z)\right|^{2}}=\frac{C^{2}(\gamma)}{\left|G_{+}(z)\right|^{2}-C^{2}(\gamma)}
$$

uniformly on each compact subset of $\overline{\mathbb{C}} \backslash \operatorname{supp}(\mu)$. Moreover, if a $\neq 1$ the numerators of (97) and (98) are different from zero on the indicated sets, thus

$$
\lim _{n \longrightarrow \infty} \frac{\phi_{n}(z) \overline{\phi_{n}(\zeta)}}{K_{n}(z, \zeta)}=\frac{G_{+}(z) \overline{G_{+}(\zeta)}-C^{2}(\gamma)}{C^{2}(\gamma)}
$$

uniformly on each compact subset of $(\overline{\mathbb{C}} \backslash \operatorname{supp}(\mu))^{2}$ and

$$
\lim _{n \longrightarrow \infty} \omega_{n}(z)\left|\phi_{n}(z)\right|^{2}=\frac{\left|G_{+}(z)\right|^{2}-C^{2}(\gamma)}{C^{2}(\gamma)},
$$

uniformly on each compact subset of $\overline{\mathbb{C}} \backslash \gamma$.

Proof. From the Christoffel formula we obtain

$$
\frac{K_{n}(z, \zeta)}{\phi_{n}(z) \overline{\phi_{n}(\zeta)}}=\frac{1}{1-z \bar{\zeta}}\left[\left(\Phi_{n+1}(0) \frac{\phi_{n}^{*}(z)}{\phi_{n}(z)}\right) \overline{\left(\Phi_{n+1}(0) \frac{\phi_{n}^{*}(\zeta)}{\phi_{n}(\zeta)}\right)} \frac{1}{\left|\Phi_{n+1}(0)\right|^{2}}-1\right] .
$$

Taking into account (37) in [4] and (86) in Corollary 1 we obtain (96). For $\mu \in M_{\Gamma}(a ; b)$ the expression on the right hand of (96) reduces to the right hand of (97). To see how this is done look at the proof of Corollary 2 and Lemma 6 in [4].

When $a_{j}=1$ for some $j \in\{1,2, \ldots, k\}$ we have $G_{-}(\xi)=0$ and $\Delta_{k}^{(j+1)}(\xi)=(z+$ $\left.b_{j+1}\right) \Delta_{k-1}^{(j+1)}(\xi)$. Thus, from (5) the right hand of (96) is

$$
\frac{1}{1-z \bar{\zeta}}\left(\frac{\left|b_{j+1}\right|^{2}}{a_{j+1}^{2}}-1\right)=0
$$




\section{References}

[1] D. Barrios, G. López, E. Torrano (1993): Location of zeros and asymptotics of polynomials satisfying three-term recurrence relations with complex coefficients. Mat. Sb., 184(11): 63-92. (English translation (1995): Russian Acad. Sci. Sb. Math., 80: 309-333).

[2] D. Barrios, G. López, E. Torrano (1995): Polynomials generated by a threeterm recurrence relation with asymptotically periodic complex coefficients. Mat. Sb. 186(5): 3-34. (English translation (1995): Sbornik: Mathematics 186(5): 629-659).

[3] D. Barrios, G. López, A. Martínez, E. Torrano: On the domain of convergence and poles of complex J-fractions. Accepted in J. of Approx. Theory.

[4] M. Bello, G. López. Ratio and relative asymptotics of polynomials orthogonal on an arc of the unit circle. Accepted in J. of Approx. Theory.

[5] A. O. Gel'fond (1967): The Calculus of Finite Differences. Moscow: Fizmatgiz (in Russian).

[6] J. S. Geronimo, W. VAn Assche (1986): Orthogonal polynomials with asymptotically periodic recurrence coefficients. J. Approx. Theory, 46: 251-283.

[7] YA. L. Geronimus (1941): On the character of the solutions of the moment problem in the case of a limit-periodic associated fraction. Izv. Akad. Nauk SSSR Ser. Mat 5: 203-210 (in Russian)

[8] Ya. L. Geronimus (1943): On polynomials orthogonal on the circle, on trigonometric moment-problem and on allied Caratheodory and Schur Functions. C.R. (Doklady), Acad. Sci. URSS, 29: 291-295.

[9] YA. L. Geronimus (1944): On polynomials orthogonal on a circle, on the trigonometric moment problem, and on the associated functions of Caratheodory's and Schur's type. Mat. Sbornik, 15(57): 99-130 (in Russian).

[10] L. Ya. Geronimus (1961): Orthogonal Polynomials. New York: Consultants Bureau.

[11] Israel Gohberg, Seymour Goldberg, Marinus A. KaAshoek (1990): Classes of Linear Operators, Vol. I. Berlin: Birkhauser.

[12] L. Golinskit, P. Nevai, W. Van Assche (1996): Perturbation of orthogonal polynomials on an arc of the unit circle. J. of Approx. Theory, 83: 392-422.

[13] P. R. Halmos (1982): A Hilbert Space Problem Book. Princeton: Van Nostrand, 2-nd Edition.

[14] T. Kato (1995): Perturbation Theory for Linear Operators. New York: SpringerVerlag.

[15] A. Máté, P. Nevai, V. Totik (1985): Asymptotics for the ratio of leading coefficients of orthonormal polynomials on the unit circle. Constructive Approximations 1: $63-69$. 
[16] P. Nevai (1979): Orthogonal Polynomials. AMS Memoires 213.

[17] F. Peherstorfer, R. Steinbauer (1996): Orthogonal polynomials on arcs of the unit circle I. J. of Approx. Theory, 85: 140-184.

[18] F. Peherstorfer, R. Steinbauer (1996): Orthogonal polynomials on arcs of the unit circle II. Orthogonal polynomials with periodic reflection coefficients. J. of Approx. Theory, 87: 60-102.

[19] F. Peherstorfer, R. Steinbauer: Asymptotic behavior for orthogonal polynomials on the unit circle with asymptotically periodic reflection coefficients. Submitted to J. of Approx. Theory.

[20] F. Peherstorfer, R. Steinbauer (1996): Comparative asymptotics for perturbed orthogonal polynomials. Trans. Amer. Math. Soc., 348: 1459-1486.

[21] E. A. Rakhmanov (1977): On the asymptotics of the ratio of orthogonal polynomials. Mat. Sb. 103(145): 237-252. (English translation (1977): Math. USSR Sb. 32: 199213.

[22] E. A. RAKhmanov (1982): On the asymptotics of the ratio of orthogonal polynomials II. Mat. Sb. 118(160): 104-117. (English translation: Math. USSR Sb. 46, 105-117).

[23] E.A. Rakhmanov (1986): On the asymptotic properties of polynomials on the circle with weights not satisfying Szegö's condition. Mat. Sb. 130(172): 151-169. (English translation: Math. USSR Sb. 58: 149-167.

[24] W. Van Assche (1992): Constructive Methods in the Analysis of Orthogonal Polynomials. Leuven: Katholieke Universiteit. 